\title{
Loss of Sarm1 non-autonomously protects Schwann cells from chemotoxicity
}

\author{
Weili Tian*, Tim Gzopka§ and Hernán López-Schier* \\ * Sensory Biology \& Organogenesis, Helmholtz Zentrum Munich, Germany \\ $\S$ Institute of Neuronal Cell Biology, Technical University of Munich, Germany
}

Correspondence to: hernan.lopez-schier@helmholtz-muenchen.de

\begin{abstract}
Abbreviated title: Sarm 1 and glioprotection
Keywords: Wallerian degeneration, Sarm1, Schwann cells, regeneration

Acknowledgements: This research was supported by a Grant from the Human Frontiers Science Programme and by the Helmholtz Gemeinschaft to H.L-S.
\end{abstract}




\section{ABSTRACT}

The obligate pro-degenerative protein Sarml is essential for Wallerian axon degeneration. Inhibition of Sarm 1 has been proposed as a promising neuroprotective strategy with clinical relevance. Yet, the conditions that will most benefit from inhibiting Sarm1 remain undefined. Here we use genetics and pharmacology in zebrafish to show that systemic elimination of Sarm1 is glioprotective. Loss of Sarm 1 does not affect macrophage recruitment to the wound microenvironment, focal injury resolution, or nerve repair. Unexpectedly, Sarm1 deficiency increases Schwann-cell resistance to toxicity by diverse chemotherapeutic agents after neuronal injury. Yet, synthetic degradation of Sarml-deficient severed axons reversed this effect, suggesting that glioprotection is non-cell-autonomous. These findings anticipate that interventions aimed at inhibiting Sarml can counter heightened glial vulnerability to chemical stressors and may be an effective strategy to reduce chronic consequences of neurotrauma. 


\section{INTRODUGTION}

1 The peripheral nerves that communicate skin, muscle and sensory organs with the

2 brain must maintain functionality throughout life despite frequent stress and trauma

3 (1-6). Loss of integrity of peripheral neurons and associated cells, including glia, is a

4 common occurrence in severe neurological dysfunctions that include weakness, pain

5 and loss of sensation (7). Glial loss leads to nerve demyelination, defasciculation and

6 neuronal death. Nerve injury triggers axon fragmentation and degeneration. In turn,

7 this induces the dedifferentiation of associated glial cells, which enhances glial role in

8 nerve repair. However, dedifferentiation also makes glia vulnerable to degeneration

9 after protracted denervation (8-12). Damaged axons undergo Wallerian

10 degeneration (13), which is regulated by the evolutionary ancient pro-degenerative

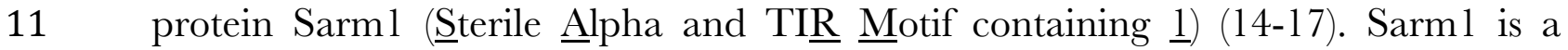

12 modular protein that contains two sterile-alpha motifs (SAM), one mitochondrial

13 association (MT) and one TIR domain (14). Nerve injury activates Sarml by TIR

14 dimerization, which is sufficient to induce the axon degeneration $(18,19)$.

15 Mechanistically, Sarml activation results in the loss of nicotinamide adenine

16 dinucleotide (NAD+) in damaged axons (20-22). The TIR domain acts as a NADase

17 (23), explaining why over-expression of the NAD+ synthesizing enzyme NMNAT1

18 inhibits the degradation of severed axons by sustaining high levels of NAD+

19 downstream of Sarm1 (24). Moreover, the loss of Sarm1 blocks the degeneration of

20 injured axons, and forced activation of Sarm1 induces axon destruction in the

21 absence of injury. Therefore, Sarml is a cell-autonomous hierarchical regulator of a

22 signaling pathway that is necessary and sufficient for axonal degradation (22). Upon 
23 peripheral-nerve injury, glial Schwann cells acquire a specialized function that

24 promotes the clearance of axon fragments ahead of axonal re-growth from the

25 proximal stump that remains associated to the neuronal perikaryon (25). Expeditious

26 axonal regeneration is important because sustained Schwann-cell denervation leads

27 to protracted loss of glial terminal phenotype and eventual death. In turn, glial loss

28 impairs regenerating nerve myelination and circuit repair, transforming acute

29 neuropathies into irreversible chronic neurological dysfunction. Therefore, inhibiting

30 or delaying axon destruction has been hypothesized as an effective strategy to counter

31 heightened Schwann-cell vulnerability to additional stressors that include metabolic

32 imbalance and drugs $(26,27)$. This idea has sparked intense efforts to identify specific

33 molecular inhibitors of axon degeneration for clinical applications (28, 29). As a

34 discrete hierarchical factor that is essential for axon degeneration, Sarml is an ideal

35 target for pharmacological interventions (18). However, the conditions that would

36 most benefit from inhibiting Sarm1 systemically are yet to be defined (30, 31$)$.

38 Here we address the above issue using the powerful genetics of zebrafish, a small 39 vertebrate whose nervous system is anatomically simpler but structurally and 40 functionally similar to that of mammals (32-34). Crucially, the zebrafish larva is ideal 41 to study Schwann-cell biology in the natural context of the behaving animal (35-42).

42 By characterizing loss-of-function mutations in Sarm1, we provide novel insights into 43 the cellular basis of axon-glia interactions, and provide data that encourage the quest 44 to identify and develop Sarm1 inhibitors for clinical applications. 


\section{RESULTS}

\section{$47 \quad$ Identification and mutagenesis of Sarm1 in zebrafish}

48 The amino-acid sequence of Sarm1 is well conserved across species $(15,43)$. To identify Sarm1 orthologs in zebrafish, we scanned publicly accessible genomic data (Danio rerio reference genome assembly version GRCz11) by a BLAST search using the TIR domain, which is present in all known Sarm1 proteins (15, 44). This exploration yielded a single candidate locus in chromosome 15. No other part of the zebrafish genome appears to harbor Sarml paralogs. The genomic structure of the putative zebrafish Sarml reminisces that of other species, containing 8 exons that code for a protein of 713 amino acids, with the typical N-terminal auto-inhibitory domain, two central SAM multimerization domains and a C-terminal TIR degeneration domain (Figure 1A). Similar to D. melanogaster, however, D. rerio Sarm 1 lacks an obvious mitochondria-targeting sequence (MT). To test whether the identified gene produces a protein with the expected functional role, we used CRISPR/Cas9-mediated genome modification to generate loss-of-function mutations in Sarm 1. By targeting exon 1, we obtained germ-line transmission of two alleles: sarm $1^{h z m 13}$ and sarm1hzm14 (Supplemental Figure 1A-B). The hzm13 allele

63 introduces an 11-base deletion and $\mathrm{T} / \mathrm{C}$ mutation, resulting in a frame shift and 64 premature stop codon. hzm14 is a 7-base deletion and AG/GA mutation that also generates a frame shift and premature stop codon. Analysis of protein extracts from wild-type embryos by Western-blot using an antibody to Sarm1 revealed a single band of approximately $80 \mathrm{kDa}$, which agrees with the expected size of the full-length protein (Supplemental Figure 1G). This band was absent in protein extracts from 
homozygous sarm $1^{\text {hzml3 }}$ zebrafish embryos. Of note, because this antibody recognizes an epitope in the C-terminus of Sarm1, it does not allow to discriminate between the expression of a truncated protein lacking all the domains with known function, and the complete absence of Sarml induced by nonsense-mediated mRNA decay. Homozygous sarm I $^{\text {zzm } 13}$ mutants display no overt anatomical defects (Supplemental Figure 1D-E), are viable and develop into fertile adults (not shown). Furthermore, a simple assay for sensorimotor function that consists of eliciting the escape response after tactile stimuli showed that the displacement distance and the average acceleration were no different between wild type and Sarm1 mutants (Supplemental Figure $1 \mathrm{~F}-\mathrm{G})(45)$.

\section{Functional conservation of Sarm1 in Wallerian axon degeneration}

81 Although Sarm 1 has already been extensively studied in neurons of Drosophila and

82 mammals, we wanted to assess the effects of systemic loss of Sarml on neuronal and 83 non-neuronal cells in larval zebrafish. The reason is that no single study has 84 addressed Sarm 1 function holistically, in a single organism, by in toto live microscopic 85 imaging. To this end, we used several parameters of nervous-system structure and 86 function by combining sarm $1^{\text {hzm } 13}$ with various transgenes expressing fluorescent 87 markers in the sensory neurons of the mechanosensory lateral line, as well as in their 88 associated Schwann cells $(46,47)$. The lateral-line system is ideal for in vivo studies at 89 high resolution over extended periods, and for physiological studies under normal 90 and altered conditions (48-51). It combines the organization of a typical vertebrate 91 sensory system with the amenability for controlled experimental interventions that 
92 include microsurgery, pharmacology and optogenetics $(41,42,52,53)$. We found

93 that loss of Sarm1 does not affect the development and maintenance of the lateral-

94 line sensory pathway (Supplemental Figure 1H-N). Next, we assessed neuronal

95 intracellular dynamics and physiological function. To this end, we fluorescently

96 marked mitochondria in lateralis neurons by expressing the mitochondria targeting

97 sequence of the Cytochrome-C oxidase subunit 8A fused to mCherry (54). We chose

98 mitochondria because mitochondria produce energy for the neurons and also

99 regulate calcium levels, which are critical for the function and viability of axons and

100 neurons. These intracellular organelles are dynamic and distribute throughout the

101 neuron by active transport mediated by molecular motors that move along

102 microtubule tracks. In axons, microtubules are polarized such that their plus ends are

103 directed toward the axon terminals, in turn orienting the movement of distinct

104 molecular motors in the antero- and retro-grade directions (55). Intra-axonal

105 movement direction also reflects mitochondrial fitness because stressed organelles are

106 biased in the retrograde direction $(56,57)$. Therefore, axonal mitochondria represent

107 an optimal proxy for neuronal polarization, intracellular dynamics and overall

108 cellular health. We found a qualitatively similar number, density and distribution of

109 mitochondria in the peripheral axons of wild type and Sarm 1 mutants. Kymographic

110 analysis revealed a majority of static large mitochondrial groups, and some smaller

111 fragments moving persistently substantial distances at constant velocity in the

112 anterograde and retrograde directions (Figure 1B-G). Importantly, quantifications

113 showed no significant differences in the number and spatial distribution of axonal 
114 mitochondria (Figure 1D), or movement velocity and direction between wild-type

115 and mutant animals (Figure 1E).

117 Next, we assayed functional conservation of Sarm 1 in this neuronal pathway using a

118 previously established bioassay of neurotrauma, which employs intact larval zebrafish,

119 laser-mediated severing individualized axons by single-neuron fluorescent-protein

120 expression, and high-resolution intravital microscopy $(41,58)$. Axotomy was done by

121 focusing an ultraviolet laser beam to a discrete region of peripheral nerve. Upon

122 severing, the distal axon segment quickly degenerates in wild-type specimens,

123 whereas the proximal segment that remains associated with the neuronal perikaryon

124 remains viable (Figure 1F). Kinetic analysis shows that severed Sarm 1-deficient axon

125 segments resisted degeneration for over 5 days. The degeneration-resistant

126 phenotype was rescued by the transgenic introduction of a fluorescent-tagged full-

127 length Sarm1 in mutant neurons (Figure 1G).

128

129 Loss of Sarm1 does not affect focal damage resolution

130 Physical injury often results in inflammatory responses that recruit immune cells to

131 the wound. After nerve injury, Schwann cells release chemoattractants for

132 macrophages before axon fragmentation is evident. Injury-mediated production of

133 reactive oxygen species (ROS) recruits inflammatory cells, including macrophages.

134 Studies ex vivo using mammalian cultured neurons showed that Sarm1 acts

135 downstream of mitochondrial ROS generation. Therefore, we decided to test in vivo

136 if the loss of Sarm 1 as well as the absence of Schwann cells would affect macrophage 
137 recruitment to the site of injury. To this end, we generated a transgenic line

138 expressing membrane-targeted EGFP under the control of the macrophage-specific

139 promoter Mfap4. We combined this line with Tg[SILL:mCherry] and mutations in

140 Sarm1 or Erbb2. Loss of Erbb2 in zebrafish impairs Schwann-cell migration along

141 lateral-line axons and leads to nerve unmyelination and defasciculation $(53,59)$. We

142 first injured nerves with the laser and assessed macrophage behavior at high

143 resolution. We found that the onset of recruitment and number of macrophages at

144 the wound did not differ between wild-type specimens and Sarm1 or Erbb2 mutants

145 (Figure 2A and Supplemental Movies 1-3). Macrophages arrived from various

146 locations moved along the proximal and distal part of the axons in wild-type and

147 mutant specimens. The retention time of macrophages at the proximal side of the

148 wound was unaltered in Sarm1 or Erbb2 mutants, although on average slightly

149 lengthened in the absence of Schwann cells (Figure 2B). In wild type, and Sarm1 and

150 Erbb2 mutants, macrophages engulfed debris locally at the injury site, and did not

151 appear to be involved in the degradation of the distal part of the severed axons

152 (Supplemental Movies 1-3). We found, however, a significant increase in the size of

153 the engulfed debris by macrophages in Erbb2-mutant animals (Figure 2C). These

154 results reveal that the loss of Sarml does not affect focal damage resolution by

155 macrophages, which are recruited to the wound independently of Schwann cells. We

156 did notice discrete and highly mobile axon fragments that were not associated to

$157 \operatorname{EGFP}(+)$ cells in wild-type and Erbb2-mutant animals, but not in Sarm1-deficient

158 fish. This observation suggests that activated phagocytic cells other than 
159 macrophages and Schwann cells engulf and clear distal axon fragments after damage,

160 and that this does not occur in Sarm1-/- animals.

161

162 Synthetic elevation of axoplasmic $\mathbf{C a}^{2+}$ induce degradation of severed

\section{Sarm1-deficient axons}

164 Calcium $\left(\mathrm{Ca}^{2+}\right)$ regulation in neurons is critical for homeostasis because sustained 165 elevation of cytosolic calcium leads to axonal and neuronal degeneration. Often, this 166 occurs because mitochondria release apoptosis-inducing factors and proteases in a 167 calcium-dependent manner (60). Studies of mammalian neurons in vitro and of 168 zebrafish have shown that neuronal damage triggers two waves of elevation of 169 axoplasmic calcium $\left(\mathrm{Ca}^{2+}\right)(21,52)$. The first occurs nearly immediately after injury 170 and decays rapidly, whereas the second has slower onset and decay. Loss of Sarm 1 171 prevents $\mathrm{Ca}^{2+}$ elevation in severed axons (21). We sought to further test the functional 172 conservation of Sarml in zebrafish in vivo by monitoring $\mathrm{Ca}^{2+}$ dynamics in lateralis 173 sensory axons at high-resolution before and after injury. We measured $\mathrm{Ca}^{2+}$ 174 dynamics in toto at high-resolution before and after axon injury using the genetically175 encoded sensor GCaMP7a, to find that intact wild-type and Sarm1-mutant axons 176 show undetectable levels of axoplasmic fluorescence above background (Figure 3A).

177 Upon severing, fluorescent signal in wild-type axons distal segments increased 178 immediately and subsequently decayed with a near constant slope, whereas 179 fluorescence remained nearly undetectable in Sarm1-deficient distal axon segments 180 (Figure 3B). Next, we examined the $\mathrm{Ca}^{2+}$ levels in mitochondria and the endoplasmic 181 reticulum (ER) using genetically encoded vital sensors, respectively Mito-RGECO 
and ER-GCaMP3 (Figure 3G-F). We selected these organelles because $\mathrm{Ca}^{2+}$ release

183 from the axonal ER activates the mitochondrial permeability transition pore to trigger axonal degeneration $(61,62)$. We found that both mitochondrial and reticular $\mathrm{Ca}^{2+}$ levels increased equally after axon severing in severed wild-type and Sarm1-/axons. These results reveal that loss of Sarml attenuates calcium influx to the axoplasm, but not $\mathrm{Ca}^{2+}$ uptake in mitochondria or the ER. microtubules and neurofilaments (63), we decided to monitor $\mathrm{Ca}^{2+}$ levels in lateralis sensory axons 2, 4, 8 and 12 hours-post-injury (hpi). In severed wild-type axons, the second wave of axoplasmic $\mathrm{Ca}^{2+}$ starts 4 hpi in coincidence with axon fragmentation, and remains elevated in axonal debris up until 8 hpi (Figure 3G). Note that the temporal resolution of these images does not allow the resolution of degeneration before axonal regeneration. By contrast, the second wave of axoplasmic $\mathrm{Ca}^{2+}$ does not occur in Sarm1-mutant axons (Figure 3H). Thus, we hypothesized that forcing a sustained elevation of axoplasmic $\mathrm{Ca}^{2+}$ will be sufficient to trigger the degradation of severed Sarm1-deficient axons. To test this prediction, we transgenically expressed the rat transient receptor potential cation channel subfamily V member 1 (TRPV1)

201 fused to tagRFP in lateralis afferent neurons of Sarm1-mutant zebrafish. Expression 202 of mCherry alone in neurons served as control. TRPV1 is non-selective cation 203 channel that exhibits a high divalent selectivity, and whose activation produces an 204 influx of $\mathrm{Ca}^{2+}$ into cells (64). Rat TRPV1 is activated by temperatures above $43^{\circ} \mathrm{C}$ 
or by the vanilloid capsaicin. Importantly, this TRPV1 is inactive at the temperature used to maintain zebrafish $\left(28^{\circ} \mathrm{C}\right)$, and zebrafish TRPV1 orthologs are insensitive to capsaicin (65). Therefore, rat TRPV1 expressed in zebrafish offers a tunable tool to elevate axoplasmic $\mathrm{Ca}^{2+}$ with excellent temporal resolution. We severed TRPV1expressing and mCherry-expressing lateralis axons, and 2 hours later a vehicle solution or vehicle + capsaicin were added in the water holding the fish

211 (Supplemental Figure 2A-B). Samples were inspected 90 minutes later. Severed Sarm1-deficient axons not expressing TRPV1 did not fragment in presence of capsaicin, or TRPV1-expressing axons bathed in ethanol solution. As hypothesized, we found that Sarm1-deficient TRPV1-expressing axon segments readily degraded in the presence of capsaicin (Supplemental Figure 2C-F). Thus, elevation of axoplasmic $\mathrm{Ca}^{2+}$ downstream of Sarm 1 is sufficient to trigger axon degradation in vivo.

\section{Schwann cells are not essential to maintain Sarm1-/- axon segments}

219 The Schwann cells support, fasciculate and myelinate sensory axons in vertebrates

220 (66). We reasoned that delayed axon degeneration by loss of Sarm 1 might impact the 221 interaction between axons and Schwann cells. To assess this interaction in vivo, we 222 combined the sarm $1^{h z m 13}$ allele with the triple transgenic line $\operatorname{Tg}[g S A G F F 202 A$; UAS223 GFP; SILL:mCherry] to highlight the Schwann cells with green fluorescence and the 224 lateralis afferent neurons with red fluorescence (53, 67). Using high-resolution 225 intravital microscopy, we ascertained that Schwann cells develop normally and 226 fasciculate sensory axons in Sarm1-deficient zebrafish (Figure 4A). Upon severing, 227 wild-type axons were quickly cleared by the Schwann cells through engulfment of 
axonal fragments and intracellular degradation of debris (Figure 4B). However, there was no axon fragmentation or phagocytic activity by the Schwann cells in Sarm 1 mutants (Figure 4G). Next, we asked if Schwann cells are necessary for the maintenance of severed Sarm1-deficient axons by generating a double mutant zebrafish line concurrently deficient for Erbb2 and Sarm1. In Erbb2-deficient specimens, the distal portion of the severed axons fragmented and were cleared (Figure 4G) but with a significant delay compared to wild-type specimens (Figure 4D). By contrast, in Erbb2/Sarm1 double mutants, severed axons did not fragment or degrade, identically to fish lacking only Sarm1 (Figure 4D). Thus, axon maintenance in Sarm 1 mutants occurs independently of the Schwann cells, and that their eventual clearance is performed by other cells.

240 When an injury generates a gap in the glia, wound-adjacent Schwann cells actively 241 move and extend cellular projections resembling filopodia to quickly reconstitute a 242 continuous glial scaffold (53). Although continuous glia are not necessary for the re243 growth of the proximal axon stump, it prevents regenerating growth cones from 244 straying, in turn facilitating end-organ de novo innervation and circuit reconstitution $245(39,41)$. Thus, we decided to interrogate Schwann-cell behavior immediately after 246 axon severing and during axon regeneration combining Tg $[g$ SAGFF202A;UAS-GFP;

247 SILL:mCherry] double transgene with the Sarm 1 mutation. As expected, we found that 248 in wild-type animals, the Schwann cells adjacent to the wound quickly extended 249 filopodia to close the gap in the glial scaffold ahead of axonal regeneration 250 (Supplemental Movie 4). Re-growing fibers then followed these sub-cellular glial 
bridges to reconstitute the nerve, suffering mild defasciculation restricted to a small area within the injury (Figure 4E and Supplemental Figure 3). In stark contrast, injury-adjacent Schwann cells did not migrate or produce filopodia-like projections in Sarml mutants (Supplemental Movie 5). Nonetheless, regenerating axons eventually negotiated the persistent larger gap to grow along the distal glial scaffold. However, the reforming nerves presented more pronounced local defasciculation (Figure 4F and Supplemental Figure 4).

\section{Loss of Sarm1 does not alter Schwann-cell phenotype after axotomy} regenerative function (70). We hypothesized that because Schwann-cell denervation would explain their lack of phagocytic and protrusive activities after axon transection.

267 Following this rationale, we immunostained samples with an antibody to Claudin-k,

268 which localizes to the junctions between mature Schwann cells and is downregulated 269 in denervated glia $(41,71)$. In wild-type specimens, Claudin-k remained strongly 270 expressed along the entire length of the lateralis afferent nerve up to 6 hours after 271 nerve injury (hpi), suggesting that distal Schwann cells remain mature during distal272 axon fragmentation (Figure 5A-B). Beginning at $10 \mathrm{hpi}$, however, distal Schwann 273 cells had markedly less Claudin-k than proximal cells. Finally, 24 hpi Glaudin-k was 
conspicuously absent from distal Schwann cells even after axons regeneration had

275 commenced. By contrast, Claudin-k remained strongly expressed after axon severing

276 in Sarml-mutant animals during the same period, with no apparent difference

277 between Schwann cells located at either side of the wound (Figure 5A,C).

278

279 Next, we assessed myelination using the 6D2 monoclonal antibody, which recognizes 280 a carbohydrate epitope in the piscine P0-like myelin glycoproteins IP1 and IP2 (72, 281 73). As with Claudin-k, 6D2 labeling faded in Schwann cells distal to the injury in wild-type specimens (Figure 6A). Yet, 6D2 labeling remained unchanged in Sarm1 mutants (Figure 6B). We obtained congruent results when addressing myelination directly in living specimens by using a transgenic line expressing membrane-targeted EGFP under the control of the myelin binding protein (Mbp:EGFP-CAAX, green) axons are maintained independently of Schwann-cell support, and that the clearance the severed axons is not necessary for regenerating axon growth, pathfinding, myelination and re-innervation of sensory organs. In addition, they reveal that Schwann cells distal to the injury do not de-differentiate in Sarm1-mutant specimens.

\section{Loss of Sarm1 counteracts Schwann-cell vulnerability to chemical toxins}

293 Many chemotherapeutic agents invariably cause peripheral neurotoxicity, leading to 294 permanent neuronal dysfunction (75-79). Under conditions of denervation, Schwann 295 cells become strongly susceptible to chemotoxicity (80). We wondered if the 296 protracted maintenance of severed axons in Sarm1 mutants may suppress glial 
vulnerability. To test this idea, we treated wild-type and Sarm1-mutant zebrafish with several chemical compounds that are under clinical trials or used as first-line treatment for common cancers in humans. First, we used 10-Hydroxycamptothecin (10-HCT), which is extremely toxic to denervated glia (82), and counted Schwann cells using the fluorescence transgenic marker $\mathrm{Tg}[g S A G F F 202 A ; U A S: E G F P]$, which is ideal for this experiment because the intensity of green fluorescence does not vary in denervated Schwann cells and, therefore, is independent of the maturity of these glia (41). We confirmed that 10-HCT does not affects in Schwann cells associated to viable axon (Figure 7A-B). However, it significantly reduced the number of Schwann cells in 10-HTC-treated wild-type animals after axon severing. By contrast, the number of distal Schwann cells was only marginally affected in Sarm1-mutant specimens (Figure 7B). Platinum-based, taxanes and some alkaloids are effective chemotherapeutic agents used as standards-of-care for various human malignancies, despite their severe neuropathic effects that include glial destruction (81). To address

316 Schwann cells from chemical stress. To confirm this prediction, we forced the 317 degradation of severed axons in Sarm1-deficient zebrafish treated with 10-HCT or 318 vincristine. To this end, we repeated the used of capsaicin to activate rat TRPV1 319 expressed in a sub-set of lateralis neurons in homozygous mutant fish, in which 
320 Sarml is absent from every cell, including Schwann cells. This experiment revealed

321 that Schwann cells lacking Sarm1 again become vulnerable to chemotoxicity once

322 severed axons were synthetically eliminated (Figure 7D), confirming that Sarm1-

323 mediated glioprotection is non-autonomous and depends upon the presence of non-

324 degradable axons.

\section{DISGUSSION}

327 The sensory neurons that innervate skin, sensory receptors, joints and muscle communicate peripheral information to the brain, enabling animals to perform the essential activities of daily life. Nerve loss has significant negative impact on the quality of life of the affected individuals. Using zebrafish and a battery of tests that

331 include subcellular structural characterization of sensory neurons and associated 332 Schwann cells, neuronal-function, and behavioral assays, we offer a comprehensive 333 and integrated analysis of neuronal and glial response to injury, as well as on the 334 consequences of blocking axon degeneration systemically. It has been well established 335 that the absence of Sarm1 in Drosophila and the mouse prevents the degradation of 336 damaged axons $(15,83)$. Although we were not predicting any differences for Sarm 1 337 in zebrafish, assaying functional conservation in our experimental neuronal pathway 338 is not dispensable because some neurotraumatic conditions had been shown to lead 339 to neuronal loss in the absence of Sarm 1. Loss of Sarm 1 improves functional recovery 340 after traumatic brain injury in mice, and inhibits vincristine-mediated neurotoxicity $341(84,85)$. Sarm1-deficient mice are viable $(19,86)$.We found that chronic and systemic 342 loss of Sarm 1 is compatible with zebrafish viability and sensorineural function, and 
demonstrate that the long-term maintenance of non-degradable axon fragments has

344 no detrimental effect on the repair of a sensory circuit. Furthermore, regenerating

345 axons fasciculate and myelinate normally, indicating that they do not compete with

346 non-degradable axons segments for exiting glia. Therefore, we conclude that the

347 axonal destructive and nerve reconstructive processes occur in parallel. In addition,

348 these data suggest that a competitive balance between axon degradation and 349 regeneration does not appear to have shaped the evolution of Sarm1 and, by 350 extension, Wallerian degeneration.

352 Focusing on Schwann cells, we show that glial cells adjacent to nerve injury in Sarm1 353 mutants behave dramatically different than those of wild-type specimens. Specifically, 354 Schwann cells in Sarm 1 mutants do not migrate or extend projections to bridge the 355 gap in the glial scaffold, indicating that these cells do not directly sense missing 356 intercellular contacts or glial discontinuity. Instead, our findings suggest alternative 357 scenarios. One is that the degradation of axons releases signals that induce Schwann 358 cell to change behavior. In mice, for example, nerve damage promotes mesenchymal 359 behavior of Schwann cells surrounding the wound via TGF- $\beta$ signaling, which drives 360 collective Schwann-cell migration across the wound (87). Although we did not 361 observe Schwann-cell migration, signals derived from injured axons may promote 362 wound-adjacent Schwann cells to extend projections to bridge the gap in a similar 363 manner. Interestingly, we observed that filopodia-like structures emerged from 364 Schwann cells at both sides of the injury in wild-type animals, suggesting that repair365 inducing signals are likely diffusible, affecting glial cells independently of their 
association with axons. It remains to be determined if the source of such signals is the

367 damaged axons, the denervated Schwann cells, or other cells in the wound 368 microenvironment.

370 Upon nerve injury, activated macrophages rapidly accumulate around the wound 371 and contribute to Wallerian degeneration and to axonal regeneration. Specifically, classical pro-inflammatory M1-type phagocytic macrophages remove axonal and myelin debris, whereas anti-inflammatory M2-type macrophages modulate Schwann-cell activity and promote axon regeneration. Immediately after nerve injury, Schwann cells release chemokines that attract or retain macrophages at the wound before axon fragmentation. Importantly, pharmacological activation of macrophage recruitment to the wound microenvironment enhances axon regeneration, whereas decrease of macrophage infiltration inhibits axon regeneration.

379 Reactive oxygen species (ROS) are conserved early wound signals across species. 380 Injury-mediated ROS production recruits inflammatory cells, including 381 macrophages. Primary sources of ROS after nerve damage are the mitochondria and 382 cellular NADPH oxidases. Studies in ex vivo cultured neurons indicate that Sarm 1 383 acts downstream of mitochondrial ROS generation. Also, previous studies have 384 characterized Wallerian axon degeneration and regeneration in Erbb2 mutant 385 specimens, but did not address effects on the wound microenvironment and the 386 behavior of macrophages (93-94). Accordingly, tested in vivo if the loss of Sarm1 as 387 well as the absence of Schwann cells would affect macrophage recruitment, to find 388 that the onset of recruitment, the retention time and number of macrophages at the 
wound did not differ between wild-type specimens and Sarm1 or Erbb2 mutants. Also, as in wild-type specimens, macrophages in Sarm1 and Erbb2 mutants engulfed debris locally, which were larger within macrophages in Ebbr2 mutants. Thus, the recruited and activated independently of the Schwann cells.

\section{GONGLUSIONS}

396 Current mechanistic details about Sarml function derive from independent observations extrapolated from a wide variety of experimental systems. This has made it difficult to synthetize findings and reconcile some conflicting observations.

399 Here, we have exploited a powerful in vivo genetic system to comprehensively study

400 the consequence of systemic loss of Sarm1 from the sub-cellular to the organismal 401 level in a single vertebrate system. By generating zebrafish carrying loss-of-function 402 mutations in Sarm1, we confirmed and deepen previous findings from Drosophila, the 403 mouse and cultured cells. Several novel insights derive from the work presented here.

404 First, that loss of Sarm1 is well tolerated by the animal. Second, that neuronal-circuit 405 repair is not contingent upon rapid clearance of damaged axons. Third, that 406 Schwann cells are not necessary for maintenance of severed axons in vivo. Fourth, that 407 after the axotomy Schwann cells distal to the cut site don't dedifferentiate in Sarm1408 mutants in vivo. Fifth, that the protracted maintenance of transected axons 409 dramatically improves Schwann-cell tolerance to chemotoxicity after nerve injury 410 after neurotrauma. This scenario is likely to occur in the clinical setting because nerve 411 trauma is an inescapable consequence of surgical interventions, chronic metabolic 
412 dysfunction including diabetes, and pharmacological treatments such as antibiotics

413 and anæsthesia that increase cellular stress (81). Therefore, these findings are of

414 obvious pathophysiological significance. Crucially, these data lend strong support to

415 the idea that direct interventions to systemically inhibit axon degradation are

416 promising strategies to reduce chronic consequences of neurotrauma. Because TIR

417 domain dimerization is necessary and sufficient to degrade NAD+, it renders Sarm 1

418 amenable to inhibition by small molecules, as it has been demonstrated for the TIR

419 domain of TLR2 (95). Our findings encourage the development of Sarm 1 inhibitors

420 for therapeutic applications $(18,29)$.

421

422

423

424

425

426

427

428

429

430

431

432

433

434

435

436

437

438 


\section{MATERIALS \& METHODS}

\section{Zebrafish strains and husbandry}

443

Zebrafish (Danio rerio) were maintained in a centralized facility in accordance to guidelines by the Ethical Committee of Animal Experimentation of the Helmholtz Zentrum München, the German Animal Welfare act Tierschutzgesetz §11, Abs. 1, number Gz.:55.2-1-54-2532-202-2014 and Gz.:55.2-2532.Vet_02-17-187 from the "Regierung von Oberbayern", Germany. The transgenic lines Tg[UAS:EGFP], Tg[MBP:tgRFP]) (89) and Erbb2 mutants $(41,59)$ have been previously published. The Tg[Sarm 1-/-] was generated by CRISPR/Cas9 mediated mutagenesis.

\section{Sarm1 mutagenesis}

We used CRISPR/Cas9-mediated genome modification to generate loss-of-function mutations in exon 1 of Sarm1. Cas9 mRNA and sgRNAs were co-injected into onecell stage embryos. Cas9 mRNA was generated in vitro from PmeI linearized CAS9 vector (pMLM3613) using the Ambion TM mMESSAGE mMACHINE T7 Kit. The mRNA was purified with the RNA easy kit (Qiagen). To generate the sgRNAs, the target exon was sequenced and the sequence information was used to design oligonucleotides for the sgRNA guide vector (pDR274) using the on-line tool "ZiFiT Targeter software package" (http://zifit.partners.org/) (90). The sgRNA sequence for exon 1 of Sarm1 is 5'-GGGACTTGGAAGAGACGCGC-3'. The annealed oligonucleotide was cloned into the BsaI-digested pDR274 vector using T4 ligase (NEB M0202). Resulting clones were sequenced to verify correctness, and then linearized with DraI. The purified linearized DNA fragment was employed to generate the sgRNA using the T7 MEGAscript kit (Ambion ${ }^{\mathrm{TM}}$ ). By out-crossing adult fish resulting from injection, we obtained germ-line transmission of two independent alleles: sarm $1^{h z m 13}$ and sarm $1^{h z m 14}$. For genotyping mutant carriers, we used the primers: 

TCAAGCAGTTTGGGAGACTC-3'.

\section{DNA constructs}

The DNA constructs SILL:mCherry, SILL:Gal4 (67), UAS:Synapsin 1-GFP (53) and mbp:EGFP-CAAX have been previously described. The vectors pMLM3613 (42251) and pDR272 (42250) were purchased from Addgene. The plasmids UAS:TRPV1tagRFP, the coding sequence of rat TRPV1 containing the E600K mutation fused to tagRFP was commercially synthesized by Genecat and the expression construct was generated using Tol2 kit. The constructs SILL:mito-mCherry, SILL:Sarm1-v2amCherry, SILL:Kaede, SILL:mitoRGECO and SILL:erGCaMP3 were generated using Tol2 kit. Mitochondria in lateralis neurons were marked by expressing the mitochondria targeting sequence from the Cytochrome-C oxidase subunit $8 \mathrm{~A}$ fused to mCherry. The plasmids containing mito-RGECO and er-GCaMP3 (92) were a gift of David W. Raible (University of Washington).

\section{Antibodies and immunostaining}

Whole-mount immunostaining was performed as following. First, samples of

zebrafish embryos or larvae were fixed by immersion in ice-cold $4 \%$ paraformaldehyde diluted into phosphate-buffered saline buffer containing $0.2 \%$ Tween20 (PBST), and incubated overnight at $4^{\circ} \mathrm{C}$. Then, the samples were washed at room temperature (RT) with PBST three times, 10 minutes per wash, and subsequently blocked in 10\% bovine serum albumin (BSA), also at RT for 1 hour. Next, the samples were incubated in primary antibodies at $4^{\circ} \mathrm{C}$ overnight. Next, the samples were washed in PBST for 2 hours, changing to fresh buffer every 30 minutes. Finally, they were incubated with secondary antibodies at $4^{\circ} \mathrm{C}$ overnight. Primary antibodies and concentrations: mouse anti-Acetylated tubulin, 1:1000 (Sigma T7251); rat anti-Claudin-k, 1:500 (gift from T. Becker, University of Edinburgh, U.K.) (71); mouse 6D2, 1:5 (gift from Dr. G. Jeserich, University of Osnabrück, Germany) (73). Secondary antibodies used were at the following concentrations: donkey anti-Mouse Alexa Fluor ${ }^{\circledR}$ 555, 1:200, Abcam ab150106; donkey anti-Rat IgG H\&L (Alexa Fluor ${ }^{\circledR}$ 647) pre-adsorbed, 1:200, Abcam ab150155). Samples were washed in PBST 
for 30 minutes and mounted in Vectashield one day before microscopic examination.

500 Imaging of fixed samples was done with a laser-scanning confocal microscope (LSM

$501 \quad 510$, Carl Zeiss).

502

\section{Intravital microscopy}

503 For videomicroscopy, larvae were anesthetized with MS-222 (0.013\% M/V) in 504 Danieau's and mounted in $0.8 \%$ low melting-point agarose on a $35 \mathrm{~mm}$ glass-bottom 505 Petri dish. Samples were gently pressed against the glass using a hair-loop glued to 506 the tip of a glass pipette, as previously described. The agarose dome was immersed 507 in Danieau's with the anesthetic. Images of the axons and Schwann cells were acquired using a spinning-disc microscope with a $40 \times$ air objective at $28.5^{\circ} \mathrm{C}(58)$. Zstacks were set to 0.8-1.2 $\mu \mathrm{m}$ intervals. Time intervals were 10 minute or 15 minute per stack. The resulting raw data were processed, assembled and analyzed with

\section{ImageJ.}

\section{Western blot assay}

513 Wild-type and mutant larvae were anesthetized and killed 5 days-post-fertilization.

514 Samples were homogenized in ice-cold RIPA buffer (with protease inhibitor cocktail 515 from Roche (Cat.04693159001). After homogenization, the samples were incubated 516 on ice for 30 minutes for further lysis. The resulting lysate was centrifuged at 1200 $517 \mathrm{rpm}$ at $4^{\circ} \mathrm{C}$, and the supernatant was taken for the BCA assay. The supernatant was 518 diluted in loading buffer and boiled at $99^{\circ} \mathrm{C}$ for 5 minutes. Next, the samples were performed in the SDS-PAGE and transferred onto a PVDF membrane. After blocking the membrane in 5\% skimmed milk (diluted in PBST) for 1 hour, the membrane was incubated with the primary antibody (rabbit anti-Sarm1, 1:500, ANASPEC 55381; Mouse anti- $\beta$-Tubulin, 1:2000, Sigma T5168) at $4^{\circ} \mathrm{C}$ overnight. The next day, the membrane was washed and incubated with HRP-labeled secondary antibody (Peroxidase-Affini Pure Goat Anti-Mouse IgG $(\mathrm{H}+\mathrm{L})$, 1:10000, Jackson Immuno Research 115035003; Peroxidase-Affini Pure Goat Anti-Rabbit $\operatorname{IgG}(\mathrm{H}+\mathrm{L})$, 1:10000, Jackson ImmunoResearch 115035144) for 1 hour. Images were acquired by developing the membrane with ECL (Pierce ${ }^{\mathrm{TM}}$ ECL Western Blotting Substrate, Thermo Fisher, 32109). 


\section{Laser microsurgery}

530 To mark lateralis sensory neurons individually, DNA of the SILL:mCherry construct 531 was injected into eggs of Tg[HGn39D], Tg[HGn39D; Sarm1-/-], Tg[SILL:mCherry; gSAGFF202A; UAS:EGFP] or Tg[SILL:mCherry ; gSAGFF202A ; UAS:EGFP ; Sarm1-/-] zebrafish. Resulting larvae were selected according to red-fluorescence in lateralis neurons. Selected samples were mounted into agarose as described above, and their peripheral axons were targeted an France), as described previously. The laser beam was delivered using a 63x waterimmersion objective $(50,53,58)$. The laser pulses were calibrated and applied to the target area until a clear gap in the axons was visible. The samples were observed again 1 hour post-injury (hpi) to confirm that the axons were completely transected.

\section{Quantification of mitochondrial density and motility}

To analyze mitochondria in sensory axons, we generated kymographs of mitomCherry fluorescent spots using the Multi-Kymograph tool of the Fiji software (http://fiji.sc). The movement of mitochondria was determined by the slope of the lines drawn over time, and the direction of movement was determined by the moving mitochondria crossing the time line (vertical) in the kymographs. The data were analyzed with Python scripts and the Graphpad Prism software.

\section{Calcium imaging}

For calcium imaging in lateralis neurons, Tg[SILL:Gal4; UAS:GCaMP7a] doubletransgenic larvae were anesthetized and mounted in $0.8 \%$ low melting-point agarose on a $35 \mathrm{~mm}$ glass-bottom Petri dish. Imaging was acquired through a $63 \mathrm{x}$ waterimmersion objective with an exposure time of 400 milliseconds. Laser-mediated axon transection was done after the $4^{\text {th }}$ imaging of the time series. Next, live videomicroscopy was done for 2 minutes at a frame rate of 400 milliseconds at $28.5^{\circ} \mathrm{C}$. The raw data were analyzed with Image J. To quantify the calcium signal, the images were processed to ImageJ. The region of interest (ROI) was selected and measured the value with time point. GCaMP or RGECO intensity changes were calculated as follows: $\Delta \mathrm{F} / \mathrm{F} 0=(\mathrm{F}-\mathrm{F} 0) / \mathrm{F} 0$, where $\mathrm{F} 0$ is the value of the fluorescent signal before 
axons were transected, and $\mathrm{F}$ is the value of the fluorescent signal with time point after axon severing (92).

\section{Chemogenetics}

562

For chemogenetic experiments, we co-injected the SILL:Gal4 with UAS:mCherry or

563 UAS:ratTRPV1-tagRFP into the Tg[UAS:GCaMP7a] or Tg[HGn39D; Sarm1-/-]. The positive larva with SILL:Gal4; UAS:ratTRPV1-tagRFP; UAS:GCaMP7a expression was used to activate TRPV1 channels in zebrafish by incubation in $5 \mu \mathrm{M}$ capsaicin (Sigma, M2028) and subsequent live imaging for 1hour of the mounted and anesthetized embryo. Images were acquired through a $63 \mathrm{x}$ water-immersion objective with an exposure time of 400 milliseconds. For experiments with the Sarm 1 mutant larvae, the HGn39D with SILL:mCherry or ratTRPV1-tagRFP positive animals were laser axotomized. The larvae were treated with $10 \mu \mathrm{M}$ capsaicin or ethanol $(1: 1000, \mathrm{v} / \mathrm{v}) 2 \mathrm{~h}$ after transection. $1.5 \mathrm{~h}$ after capsaicin treatment, images were taken by spinning disc microscopy.

\section{Behavioral assays}

574 For the touch-mediated escape response, 2dpf embryos were gently dechorionated and kept in Danieau's solution at $28^{\circ} \mathrm{C}$ for at least 1 hour. Embryos were place into a flat uncovered Petri dish containing Danieau's and were recorded with a high-speed camera (NX4 series, Imaging solution, GmbH). Video recording was launched and a randomly chosen embryo was touched with a blunt glass needle until it evoked a reaction. Recordings were done under white-light illumination over 150 seconds at a rate of 200 frames per second (fps). The swimming trajectories were obtained with 3D Particle Tracker plugin, ImageJ software. The further quantification and statistics

\section{3} were using Python.

\section{Statistical analysis}




\section{REFERENGES}

591

592 1. Ameh V, and Crane S. Nerve injury following shoulder dislocation: the

593

594

595

596

597

598

599

600

601

602

603

604

605

606

607

608

609

610

611

612

613

614

615

616

617

618

619 emergency physician's perspective. Eur 7 Emerg Med. 2006;13(4):233-5.

2. Heneka MT, Carson MJ, El Khoury J, Landreth GE, et al. Neuroinflammation in Alzheimer's disease. Lancet Neurol. 2015;14(4):388-405.

3. Missios S, Bekelis K, and Spinner RJ. Traumatic peripheral nerve injuries in

children: epidemiology and socioeconomics. 7 Neurosurg Pediatr. 2014;14:688-94.

4. Rowe RK, Ellis GI, Harrison JL, Bachstetter AD, et al. Diffuse traumatic brain injury induces prolonged immune dysregulation and potentiates hyperalgesia following a peripheral immune challenge. Mol Pain. 2016;12.

5. Tzekov R, Phifer J, Myers A, Mouzon B, and Crawford F. Inflammatory changes in optic nerve after closed-head repeated traumatic brain injury:

Preliminary study. Brain Inj. 2016;30(12):1428-35.

6. Ziogas NK, and Koliatsos VE. Primary Traumatic Axonopathy in Mice Subjected to Impact Acceleration: A Reappraisal of Pathology and Mechanisms with High-Resolution Anatomical Methods. 7 Neurosci. 2018; 38:4031-47.

7. Roselli F, and Caroni P. From intrinsic firing properties to selective neuronal vulnerability in neurodegenerative diseases. Neuron. 2015;85(5):901-10.

8. Cattin AL, and Lloyd AC. The multicellular complexity of peripheral nerve regeneration. Curr Opin Neurobiol. 2016;39:38-46.

9. Parrinello S, Napoli I, Ribeiro S, Wingfield Digby P, Fedorova M, Parkinson DB, et al. EphB signaling directs peripheral nerve regeneration through Sox2dependent Schwann cell sorting. Cell. 2010;143(1):145-55.

10. Ziegenfuss JS, Doherty J, and Freeman MR. Distinct molecular pathways mediate glial activation and engulfment of axonal debris after axotomy. $\mathcal{N a t}$ Neurosci. 2012;15(7):979-87.

11. Harrisingh MC, Pérez-Nadales E, Parkinson DB, Malcolm DS, Mudge AW, and Lloyd AC. The Ras/Raf/ERK signalling pathway drives Schwann cell dedifferentiation. EMBO 7. 2004;23(15):3061-71.

12. Scherer SS. The biology and pathobiology of Schwann cells. Curr Opin Neurol. 1997;10(5):386-97.

13. Waller A. Experiments on the Section of the Glossopharyngeal and Hypoglossal Nerves of the Frog, and Observations of the Alterations Produced Thereby in the Structure of Their Primitive Fibres. Philosophical Transactions of the Royal Society of London. 140:423-9

14. Gerdts J, Summers DW, Milbrandt J, and DiAntonio A. Axon Self- 
646

647

648

649

650

651

652

653

654

655

656

657

658

659

660

661

662

663

664

665

666

667

668

669

670

671

672

673

674

675

676

677

16. Walker LJ, Summers DW, Sasaki Y, Brace EJ, Milbrandt J, and DiAntonio A. MAPK signaling promotes axonal degeneration by speeding the turnover of the axonal maintenance factor NMNAT2. Elife. 2017;6.

17. Yang J, Wu Z, Renier N, Simon DJ, et al. Pathological axonal death through a MAPK cascade that triggers a local energy deficit. Cell. 2015;160:161-76.

18. Essuman K, Summers DW, Sasaki Y, Mao X, DiAntonio A, and Milbrandt J. The SARM1 Toll/Interleukin-1 Receptor Domain Possesses Intrinsic NAD(+) Cleavage Activity that Promotes Pathological Axonal Degeneration. Neuron. 2017;93(6):1334-43 e5.

19. Gerdts J, Summers DW, Sasaki Y, DiAntonio A, and Milbrandt J. Sarm1mediated axon degeneration requires both SAM and TIR interactions. $\mathcal{F}$ Neurosci. 2013;33(33):13569-80.

20. Gerdts J, Brace EJ, Sasaki Y, DiAntonio A, and Milbrandt J. SARM1 activation triggers axon degeneration locally via $\mathrm{NAD}(+)$ destruction. Science. 2015;348(6233):453-7.

21. Loreto A, Di Stefano M, Gering M, and Conforti L. Wallerian Degeneration Is Executed by an NMN-SARM1-Dependent Late $\mathrm{Ca}(2+)$ Influx but Only Modestly Influenced by Mitochondria. Cell Rep. 2015;13(1 1):2539-52.

22. Summers DW, Gibson DA, DiAntonio A, and Milbrandt J. SARM1-specific motifs in the TIR domain enable NAD+ loss and regulate injury-induced SARM1 activation. Proc Natl Acad Sci U S A. 2016;113(41):E6271-E80.

23. Essuman K, Summers DW, Sasaki Y, Mao X, Yim AKY, DiAntonio A, et al. TIR Domain Proteins Are an Ancient Family of NAD(+)-Consuming Enzymes. Curr Biol. 2018;28(3):421-30 e4.

24. Sasaki Y, Nakagawa T, Mao X, DiAntonio A, and Milbrandt J. NMNAT1 inhibits axon degeneration via blockade of SARM1-mediated $\mathrm{NAD}(+)$ depletion. Elife. 2016;5.

25. Jessen KR, Mirsky R, and Lloyd AG. Schwann Cells: Development and Role in Nerve Repair. Cold Spring Harb Perspect Biol. 2015;7(7):a020487.

26. Jortner BS. Mechanisms of toxic injury in the peripheral nervous system: neuropathologic considerations. Toxicol Pathol. 2000;28(1):54-69.

27. Morell P, and Toews AD. Schwann cells as targets for neurotoxicants. Neurotoxicology. 1996;17(3-4):685-95.

28. Koeppen AH. Wallerian degeneration: history and clinical significance. $\mathcal{F}$ Neurol Sci. 2004;220(1-2):115-7.

29. Simon DJ, and Watkins TA. Therapeutic opportunities and pitfalls in the treatment of axon degeneration. Curr Opin Neurol. 2018;31(6):693-701.

30. Schuldiner $\mathrm{O}$, and Yaron A. Mechanisms of developmental neurite pruning. Cell Mol Life Sci. 2015;72(1):101-19.

31. Omura T, Omura K, Tedeschi A, Riva P, et al. Robust Axonal Regeneration Occurs in the Injured CAST/Ei Mouse CNS. Neuron. 2015;86(5):1215-27.

32. D'Rozario M, Monk KR, and Petersen SC. Analysis of myelinated axon formation in zebrafish. Methods Cell Biol. 2017;138:383-414.

33. Friedrich RW, Genoud C, and Wanner AA. Analyzing the structure and function of neuronal circuits in zebrafish. Front Neural Circuits. 2013;7:71. 
34. Friedrich RW, Jacobson GA, and Zhu P. Circuit neuroscience in zebrafish. Curr Biol. 2010;20(8):R371-81.

35. Ackerman SD, Garcia C, Piao X, Gutmann DH, and Monk KR. The adhesion GPCR Gpr56 regulates oligodendrocyte development via interactions with Galpha12/13 and RhoA. Nat Commun. 2015;6:6122.

36. Bin JM, and Lyons DA. Imaging Myelination In Vivo Using Transparent Animal Models. Brain Plast. 2016;2(1):3-29.

37. Fontenas L, and Kucenas S. Livin' On The Edge: glia shape nervous system transition zones. Curr Opin Neurobiol. 2017;47:44-51.

38. Ohki Y, Wenninger-Weinzierl A, Hruscha A, Asakawa K, Haass C, et al. Glycine-alanine dipeptide repeat protein contributes to toxicity in a zebrafish model of C9orf72 associated neurodegeneration. Mol Neurodegener. 2017;12(1):6.

39. Rosenberg AF, Isaacman-Beck J, Franzini-Armstrong C, and Granato M. Schwann cells and deleted in colorectal carcinoma direct regenerating motor axons towards their original path. F Neurosci. 2014;34(44):14668-81.

40. Wehner D, Tsarouchas TM, Michael A, Haase C, Weidinger G, Reimer MM, et al. Wnt signaling controls pro-regenerative Collagen XII in functional spinal cord regeneration in zebrafish. Nat Commun. 2017;8(1):126.

41. Xiao Y, Faucherre A, Pola-Morell L, Heddleston JM, Liu TL, Chew TL, et al. High-resolution live imaging reveals axon-glia interactions during peripheral nerve injury and repair in zebrafish. Dis Model Mech. 2015;8(6):553-64.

42. O'Donnell KC, Vargas ME, and Sagasti A. WldS and PGC-1 alpha regulate mitochondrial transport and oxidation state after axonal injury. $\mathcal{F}$ Neurosci. 2013;33(37):14778-90.

43. Conforti L, Gilley J, and Coleman MP. Wallerian degeneration: an emerging axon death pathway linking injury and disease. Nat Rev Neurosci. 2014;15:394.

44. Howe K, Clark MD, Torroja CF, Torrance J, Berthelot C, Muffato M, et al. The zebrafish reference genome sequence and its relationship to the human genome. Nature. 2013;496(7446):498-503.

45. Sztal TE, Ruparelia AA, Williams C, and Bryson-Richardson RJ. Using Touch-evoked Response and Locomotion Assays to Assess Muscle Performance and Function in Zebrafish. $\mathcal{F}$ Vis Exp. 2016(116).

46. Ghysen A, and Dambly-Chaudière C. The lateral line microcosmos. Genes Dev. 2007;21(17):2118-30.

47. Pujol-Martí J, and López-Schier H. Developmental and architectural principles of the lateral-line neural map. Front Neural Circuits. 2013;7:47.

48. Drerup GM, and Nechiporuk AV. In vivo analysis of axonal transport in zebrafish. Methods Cell Biol. 2016;131:311-29.

49. Haehnel-Taguchi M, Akanyeti O, and Liao JC. Behavior, Electrophysiology, and Robotics Experiments to Study Lateral Line Sensing in Fishes. Integr Comp Biol. 2018;58(5):874-83.

50. Pujol-Martí J, Faucherre A, Aziz-Bose R, Asgharsharghi A, Colombelli J, Trapani JG, et al. Converging axons collectively initiate and maintain synaptic selectivity in a constantly remodeling sensory organ. Curr. Biol. 2014;24(24):2968-74. 
51. Olt J, Ordoobadi AJ, Marcotti W, and Trapani JG. Physiological recordings from the zebrafish lateral line. Methods Cell Biol. 2016;133:253-79.

52. Vargas ME, Yamagishi Y, Tessier-Lavigne M, and Sagasti A. Live Imaging of Calcium Dynamics during Axon Degeneration Reveals Two Functionally Distinct Phases of Calcium Influx. F Neurosci. 2015;35(45):15026-38.

53. Xiao Y, Tian W, and Lopez-Schier H. Optogenetic stimulation of neuronal repair. Curr Biol. 2015;25(22):R1068-9.

54. Kim MJ, Kang KH, Kim CH, and Choi SY. Real-time imaging of mitochondria in transgenic zebrafish expressing mitochondrially targeted GFP. Biotechniques. 2008;45(3):331-4.

55. Mandal A, Pinter K, and Drerup CM. Analyzing Neuronal Mitochondria in vivo Using Fluorescent Reporters in Zebrafish. Front Cell Dev Biol. 2018;6:144.

56. Lin MY, Cheng XT, Tammineni P, Xie Y, Zhou B, Cai Q et al. Releasing Syntaphilin Removes Stressed Mitochondria from Axons Independent of Mitophagy under Pathophysiological Conditions. Neuron. 2017;94:595-610 e6.

57. Miller KE, and Sheetz MP. Axonal mitochondrial transport and potential are correlated. $\mathcal{F}$ Cell Sci. 2004;117(Pt 13):2791-804.

58. Xiao Y, and López-Schier H. Studying Axonal Regeneration by Laser Microsurgery and High-Resolution Videomicroscopy. Methods Mol Biol. 2016;1451:271-80.

59. Lyons DA, Pogoda HM, Voas MG, Woods IG, Diamond B, Nix R, et al. erbb3 and erbb2 are essential for schwann cell migration and myelination in zebrafish. Curr Biol. 2005;15(6):513-24.

60. Ozaki T, Yamashita T, and Ishiguro S. Mitochondrial m-calpain plays a role in the release of truncated apoptosis-inducing factor from the mitochondria. Biochim Biophys Acta. 2009;1793(12):1848-59.

61. Court FA, and Coleman MP. Mitochondria as a central sensor for axonal degenerative stimuli. Trends Neurosci. 2012;35(6):364-72.

62. Villegas R, Martinez NW, Lillo J, Pihan P, Hernandez D, Twiss JL, et al. Calcium release from intra-axonal endoplasmic reticulum leads to axon degeneration through mitochondrial dysfunction. F Neurosci. 2014;34:7179-89.

63. Park JY, Jang SY, Shin YK, Suh DJ, and Park HT. Calcium-dependent proteasome activation is required for axonal neurofilament degradation. Neural Regen Res. 2013;8(36):3401-9.

64. Caterina MJ, Schumacher MA, Tominaga M, Rosen TA, Levine JD, and Julius D. The capsaicin receptor: a heat-activated ion channel in the pain pathway. Nature. 1997;389(6653):816-24.

65. Gau P, Poon J, Ufret-Vincenty C, Snelson CD, Gordon SE, Raible DW, et al. The zebrafish ortholog of TRPV1 is required for heat-induced locomotion. $\mathcal{F}$ Neurosci. 2013;33(12):5249-60.

66. Brosius LA, and Barres BA. Contrasting the glial response to axon injury in the central and peripheral nervous systems. Dev. Cell. 2014;28(1):7-17.

67. Faucherre A, Pujol-Martí J, Kawakami K, and López-Schier H. Afferent neurons of the zebrafish lateral line are strict selectors of hair-cell orientation. PLoS One. 2009;4(2):e4477. 
68. Sasaki Y, Hackett AR, Kim S, Strickland A, and Milbrandt J. Dysregulation of $\mathrm{NAD}(+)$ Metabolism Induces a Schwann Cell Dedifferentiation Program. $\mathcal{J}$ Neurosci. 2018;38(29):6546-62.

773

774

69. Soto J, and Monje PV. Axon contact-driven Schwann cell dedifferentiation. Glia. 2017;65(6):864-82.

70. Carr MJ, and Johnston AP. Schwann cells as drivers of tissue repair and regeneration. Curr Opin Neurobiol. 2017;47:52-7.

71. Munzel EJ, Schaefer K, Obirei B, Kremmer E, Burton EA, Kuscha V, et al. Claudin $\mathrm{k}$ is specifically expressed in cells that form myelin during development of the nervous system and regeneration of the optic nerve in adult zebrafish. Glia. 2012;60(2):253-70.

72. López-Schier H, and Hudspeth AJ. Supernumerary neuromasts in the posterior lateral line of zebrafish lacking peripheral glia. Proc Natl Acad Sci U S A. 2005; 102(5):1496-501.

73. Bastmeyer M, Jeserich G, and Stuermer CA. Similarities and differences between fish oligodendrocytes and Schwann cells in vitro. Glia. 1994;11:300-14.

74. Czopka T, Ffrench-Constant C, and Lyons DA. Individual oligodendrocytes have only a few hours in which to generate new myelin sheaths in vivo. Dev Cell. 2013;25(6):599-609.

75. Cavaletti G, Alberti P, and Marmiroli P. Chemotherapy-induced peripheral neurotoxicity in the era of pharmacogenomics. Lancet Oncol. 2011;12:1151-61.

76. Kaiser J, Bledowski C, and Dietrich J. Neural correlates of chemotherapyrelated cognitive impairment. Cortex. 2014;54:33-50.

77. McLeary F, Davis A, Rudrawar S, Perkins A, and Anoopkumar-Dukie S. Mechanisms underlying select chemotherapeutic-agent-induced neuroinflammation and subsequent neurodegeneration. Eur 7 Pharmacol. 2018;842:49-56.

78. Fukuda Y, Li Y, and Segal RA. A Mechanistic Understanding of Axon Degeneration in Chemotherapy-Induced Peripheral Neuropathy. Front Neurosci. 2017;11:481.

79. Gornstein E, and Schwarz TL. The paradox of paclitaxel neurotoxicity: Mechanisms and unanswered questions. Neuropharmacology. 2014;76:175-83.

80. Imai S, Koyanagi M, Azimi Z, Nakazato Y, Matsumoto M, Ogihara T, et al. Taxanes and platinum derivatives impair Schwann cells via distinct mechanisms. Sci Rep. 2017;7(1):5947.

81. Yang S, Abrahams MS, Hurn PD, Grafe MR, Kirsch JR. Local anesthetic Schwann cell toxicity is time and concentration dependent. Reg Anesth Pain Med. 2011;36:444-51.

82. Bremer J, Skinner J, and Granato M. A small molecule screen identifies in vivo modulators of peripheral nerve regeneration in zebrafish. PLoS One. 2017;12(6):e0178854.

83. Gilley J, Ribchester RR, and Coleman MP. Sarm1 Deletion, but Not Wld(S), Confers Lifelong Rescue in a Mouse Model of Severe Axonopathy. Cell Rep. 2017;21:10-6. 
84. Henninger N, Bouley J, Sikoglu EM, An J, Moore CM, King JA, et al. Attenuated traumatic axonal injury and improved functional outcome after traumatic brain injury in mice lacking Sarm1. Brain. 2016;139(Pt 4):1094-105.

85. Geisler S, Doan RA, Strickland A, Huang X, Milbrandt J, and DiAntonio A. Prevention of vincristine-induced peripheral neuropathy by genetic deletion of SARM1 in mice. Brain. 2016;139(Pt 12):3092-108.

86. Turkiew E, Falconer D, Reed N, and Hoke A. Deletion of Sarml gene is neuroprotective in two models of peripheral neuropathy. 7 Peripher Nero Syst. 2017;22(3):162-71.

87. Clements MP, Byrne E, Camarillo Guerrero LF, Cattin AL, Zakka L, Ashraf A, et al. The Wound Microenvironment Reprograms Schwann Cells to Invasive Mesenchymal-like Gells to Drive Peripheral Nerve Regeneration. Neuron. 2017;96(1):98-114 e7.

88. Muto A, Ohkura M, Abe G, Nakai J, and Kawakami K. Real-time visualization of neuronal activity during perception. Curr Biol. 2013;23:307-11.

89. Auer F, Vagionitis S, and Czopka T. Evidence for Myelin Sheath Remodeling in the CNS Revealed by In Vivo Imaging. Curr Biol. 2018;28(4):549-59 e3.

90. Wright DA, Thibodeau-Beganny S, Sander JD, Winfrey RJ, Hirsh AS, Eichtinger M, et al. Standardized reagents and protocols for engineering zinc finger nucleases by modular assembly. Nat Protoc. 2006;1(3):1637-52.

91. Czopka $\mathrm{T}$, and Lyons DA. Individual oligodendrocytes have only a few hours in which to generate new myelin sheaths in vivo. Dev. Cell. 2013;25(6):599-609.

92. Esterberg R, Linbo T, Pickett SB, Wu P, Ou HC, Rubel EW, et al. Mitochondrial calcium uptake underlies ROS generation during aminoglycoside-induced hair cell death. F Clin Invest. 2016;126(9):3556-66.

93. Atanasoski, S., Scherer, S. S., Sirkowski, E., Leone, D., Garratt, A. N., Birchmeier, C., \& Suter, U. ErbB2 signaling in Schwann cells is mostly dispensable for maintenance of myelinated peripheral nerves and proliferation of adult Schwann cells after injury. F. Neurosci. 2006; 26: 2124-31.

94. Han, S. B., Kim, H., Lee, H., Grove, M., Smith, G. M., \& Son, Y. J. Postinjury induction of activated ErbB2 selectively hyperactivates denervated Schwann cells and promotes robust dorsal root axon regeneration. F. Neurosci. 2017; 37: 10955-10970.

95. Mistry P, Laird MH, Schwarz RS, Greene S, Dyson T, Snyder GA, et al. Inhibition of TLR2 signaling by small molecule inhibitors targeting a pocket within the TLR2 TIR domain. Proc. Natl. Acad. Sci. U.S.A. 2015; 112:5455-60. 
A

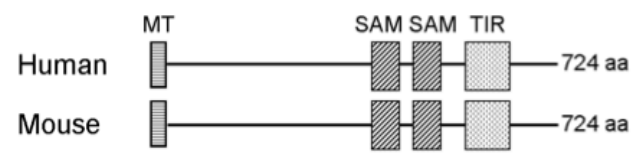

B

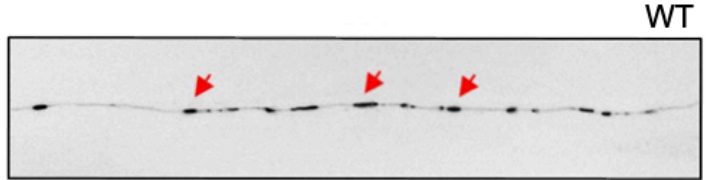

C

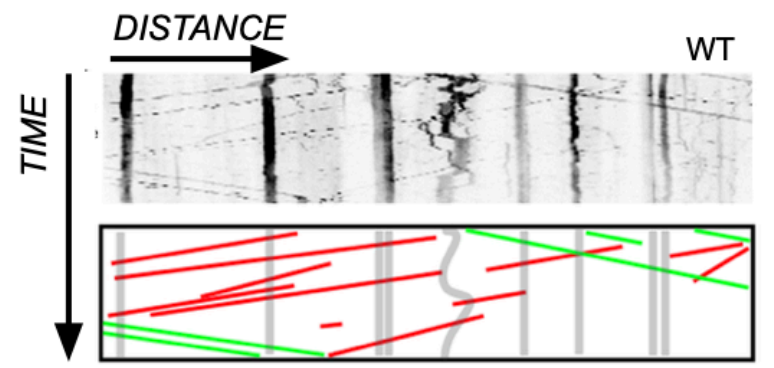

D

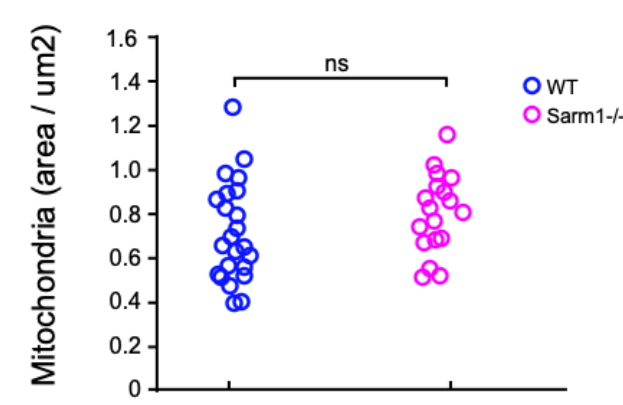

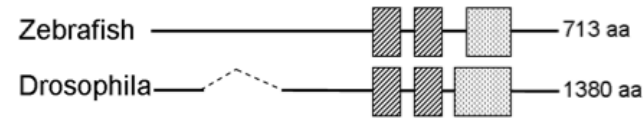

Sarm1-/-

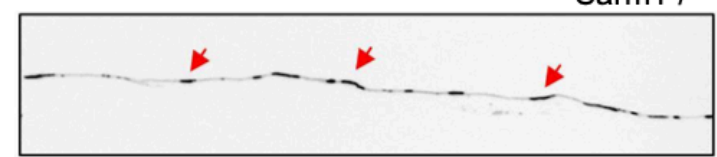

Sarm1-/-

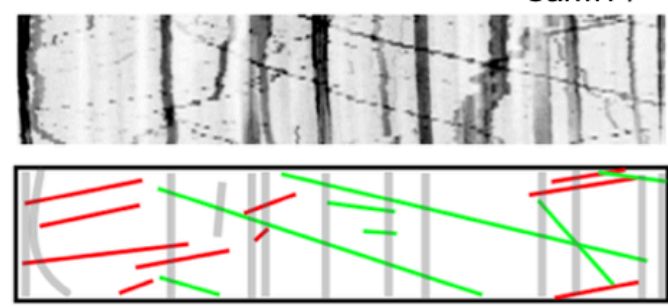

E

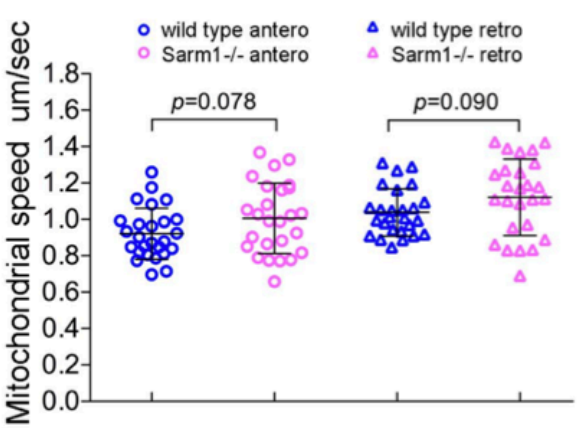

$\mathrm{F}$
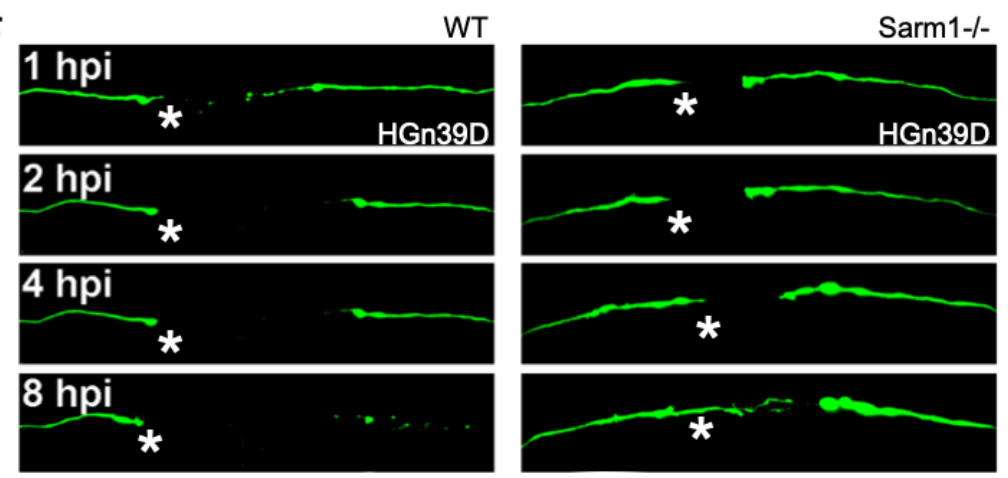

G

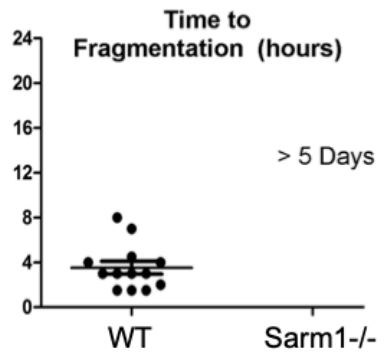

Figure 1. A) Structure Sarm 1 indicating alignment of the Sarm 1 functional domains from different species (not at scale). B) Confocal image of axonal mitochondria marked with mito-mCherry in wild-type and Sarm1-/-. Red arrows point to prominent mitochondrial groups in axons. C) Upper panels, kymographs from videomicroscopic recording of axonal mitochondria in wild type $(\mathrm{H})(\mathrm{left}$ panel) and 
861 Sarm1-/- (I) (right panel). Lower panels show color-coded traces of moving 862 mitochondria in anterograde (green) and retrograde (red) directions, taken from the

863 kymographs shown in the upper panels. D) Density of mitochondria in 5dpf wild 864 type and Sarm 1-/-, Error bar = SEM; n.s. = not significant, p value from Student's 865 t test, n=25 (WT), n=19 (Sarm1-/-). E) Mobility of the mitochondria in 5dpf wild 866 type and Sarm1-/-. Circles show the anterograde and triangles the retrograde 867 movement of the mitochondria. p value from one-way ANOVA, wild type $n=26$, 868 Sarm1-/- n=26. F) Time-lapse images of axonal degeneration of GFP-labeled 869 lateralis sensory neuron in wild-type (left) and Sarm1-/- larvae (right). hpi = hour 870 post injury, scale bar $=50 \mu \mathrm{m}$, white asterisk indicates the regrowing axons from the 871 proximal stump. G) Quantification of the time from axon transection to 872 fragmentation in wild type $(n=13)$ and Sarm1-/- $(n=13)$.

873

874

875

A

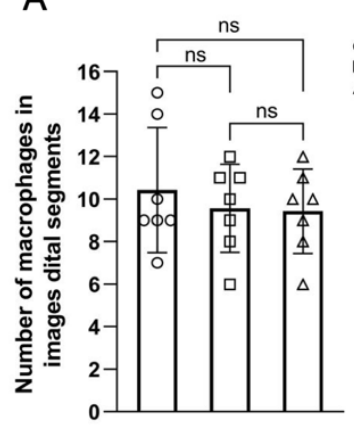

B

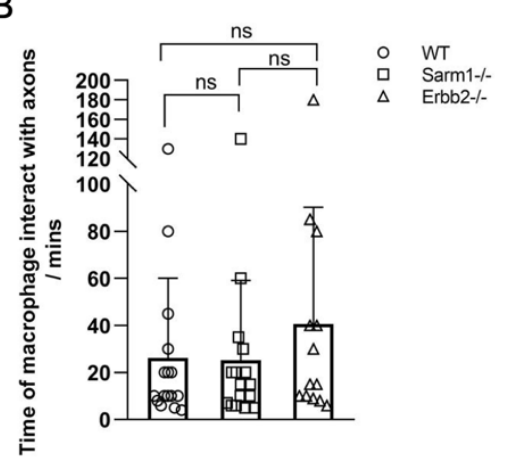

C

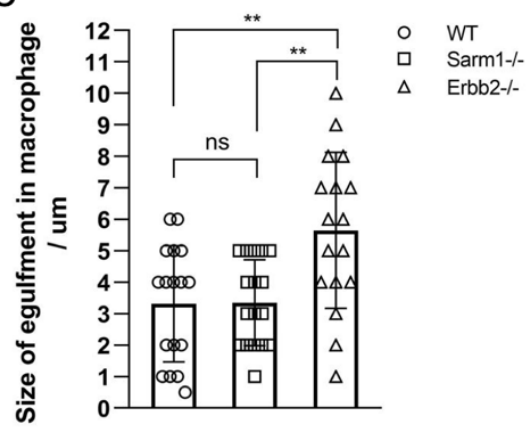

876 Figure 2. A) Quantification of the number of macrophages recruited to the injury

877 site and adjacent axon segments. B) Quantification of the time macrophages interact 878 with axon segments. G) Quantification of the size of debris within macrophages. P 879 values for T-test. 
A

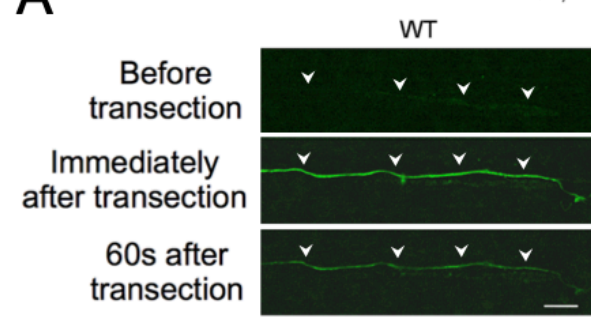

C

$$
\begin{gathered}
\text { Before } \\
\text { transection } \\
\text { Immediately } \\
\text { after transection } \\
60 \text { s after } \\
\text { transection }
\end{gathered}
$$

E $\begin{gathered}\text { Before } \\ \text { transection }\end{gathered}$

Immediately after transection

60s after transection
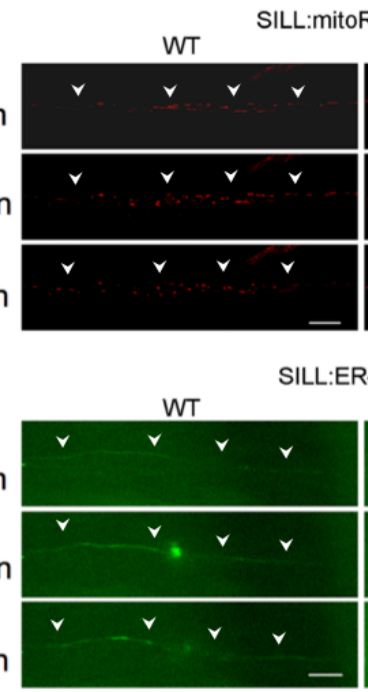
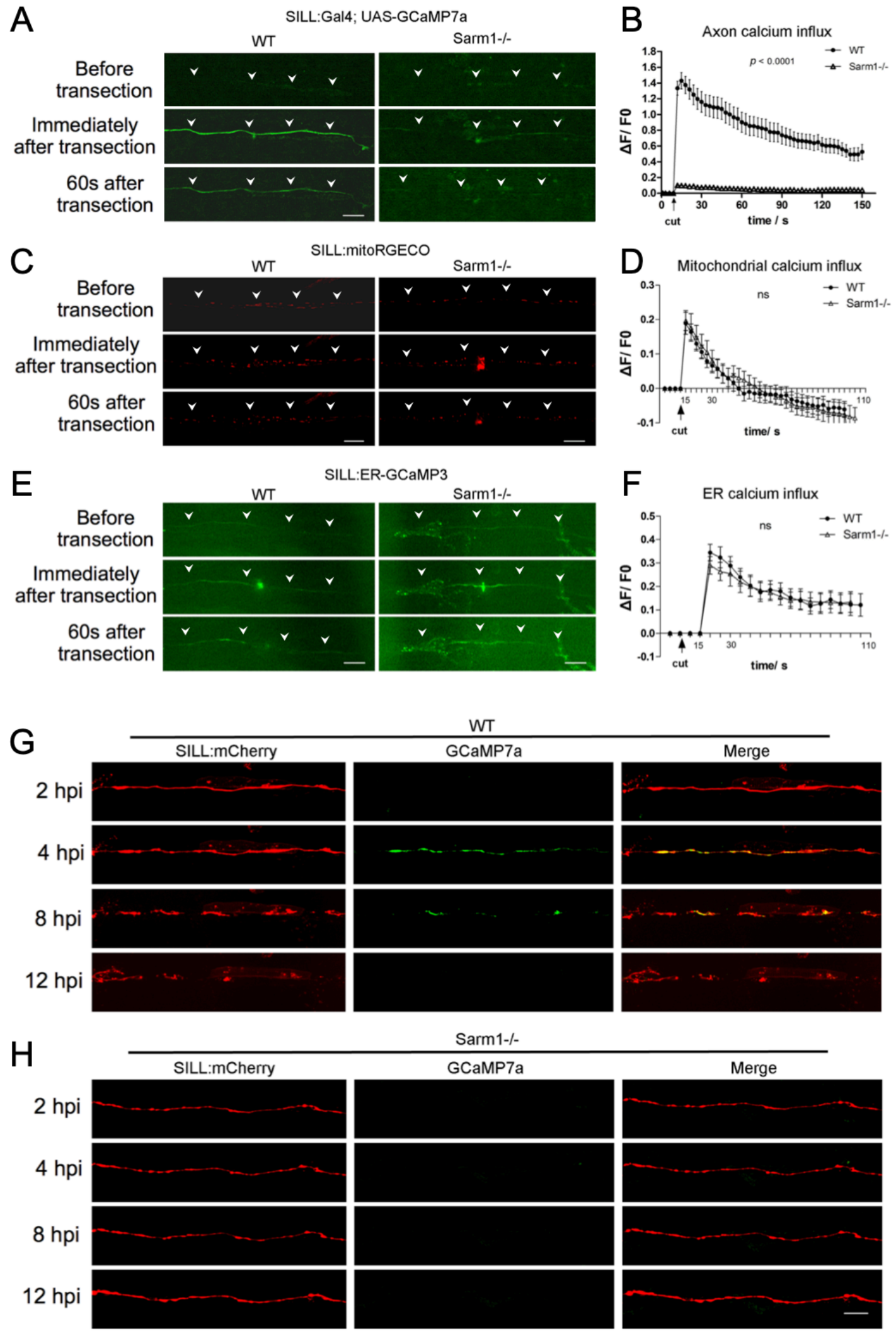

Sarm1-/-
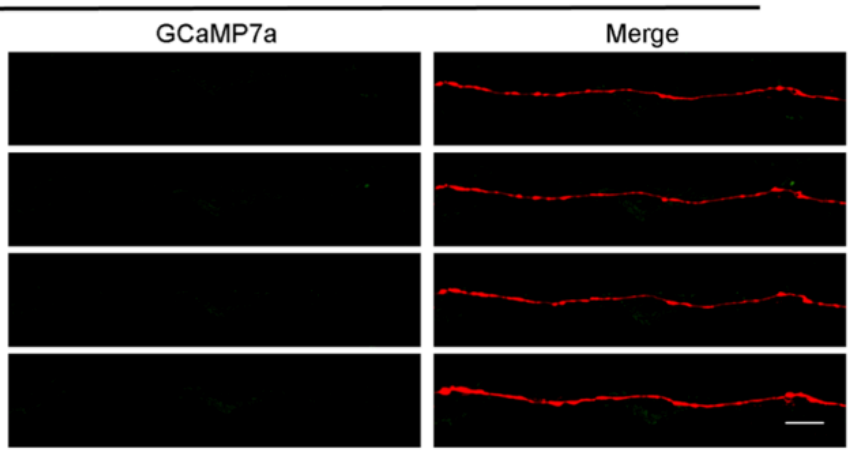

Figure 3. A) Confocal image of a single lateralis sensory axon expressing the green- 
(right column). Rows show that same samples before laser-mediated transection (top), immediately after transection (middle) and 60 seconds after transection (bottom). In A, C and E, white arrowheads indicate the position of the axon, specifically when

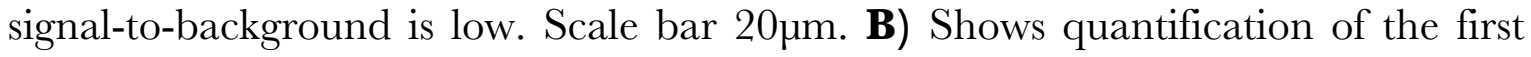
wave of axoplasmic calcium. Data are shown as mean \pm SEM; p from one-way ANOVA, wild type $n=16$, Sarm1-/- 16. G) Shows a confocal image of lateralis sensory axons expressing the red-fluorescent calcium sensor RGECO in wild type (left column) and Sarm1-/- fish (right column). Rows show that same samples before laser-mediated transection (top), immediately after transection (middle), and 60 seconds after transection (bottom). D) Quantification mitochondrial calcium influx shows the strong and nearly identical elevation and decay in wild type and Sarm 1-/immediately after the cuts. Data are shown as mean \pm SEM; p from one-way ANOVA, wild type $n=16$, Sarm1-/- 16. E) Shows a confocal image of lateralis sensory axons expressing the green-fluorescent calcium sensor CCaMP3 targeted to the endoplasmic reticulum (ER) in wild type (left column) and Sarm1-/- fish (right column). Rows show that same samples before laser-mediated transection (top), immediately after transection (middle), and 60 seconds after transection (bottom). F) Quantification ER calcium influx shows strong and statistically equal elevation and decay in wild type and Sarm1-/- after the cuts. Data are shown as mean \pm SEM; p lateralis sensory axons expressing the mCherry (red) and green-fluorescent calcium sensor GCaMP7a (green) in wild type fish. Rows show same axons 2, 4, 8 and 12 hours after transection (hours-post-injury $=$ hpi). H) Confocal image of lateralis 


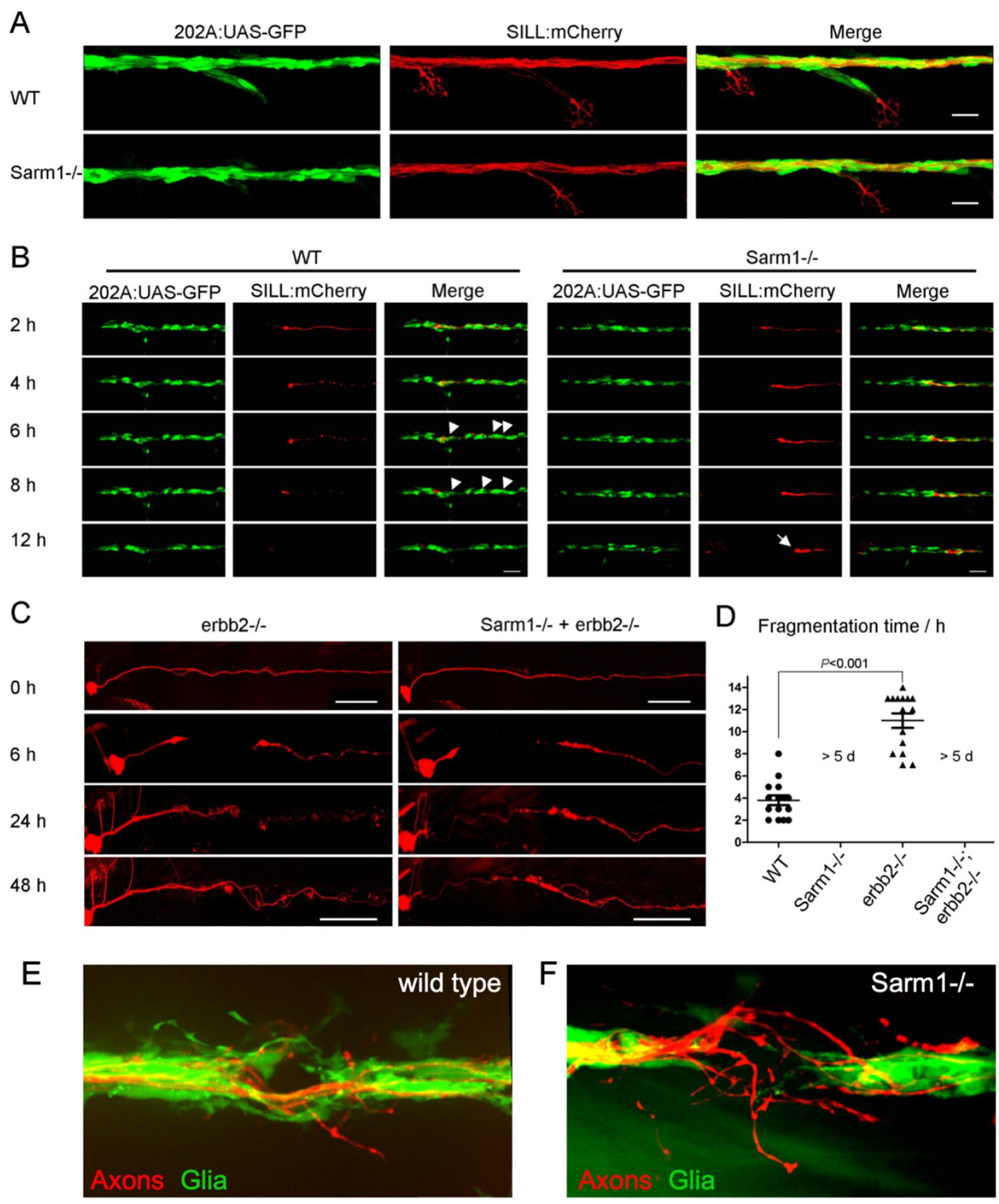

Figure 4. A) Confocal images of a double transgenic 5dpf larva showing Schwann

912 cells marked by expression of GFP (green) under the control of the

$913 \mathrm{Tg}$ [gSAGFF202A] Gal4 driver, and lateralis afferent neurons marked by expression

914 of mCherry under the control of the SILL enhancer (red). Wild type (top), Sarm 1

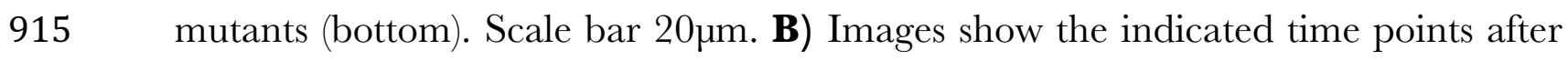

916 axon transection (hours post injury $=$ hpi) from a videomicroscopic recording of

917 Schwann cells (green) and their interaction with axons (red) in wild-type and Sarm1-

918 /-. White arrowheads indicate Schwann cells engulfing axonal debris in the wild type. 
A white arrow indicates degradation-resistant axon segment in Sarm1-/-. Please, note that the proximal axon stump in Sarm1-/- is not visible in these images because it is outside the focal plane. $\mathbf{C}$ ) Images of mCherry-expressing (red) transected axons in Erbb2-/- mutants and Sarm 1-/- ; ErBb2-/- double mutants. Scale bar 100um. D) Quantification of transected axon fragmentation in Erbb2-/- and Sarm1-/-; ErBb2/.. Error bar = SEM, p value from One-way ANOVA test, $\mathrm{n}=15$ (each group). E) Image of a from Supplemental Movie 1, showing the discrete local defasciculation of regenerated the sensory fiber (red) and the bridging of the glial gap by Schwann cells (green) in a wild-type specimen. F) Equivalent experiment, taken from Supplemental sensory fiber (red) in a Sarm1-mutant specimen. Note that and the bridging of the glial gap does not occur.

A
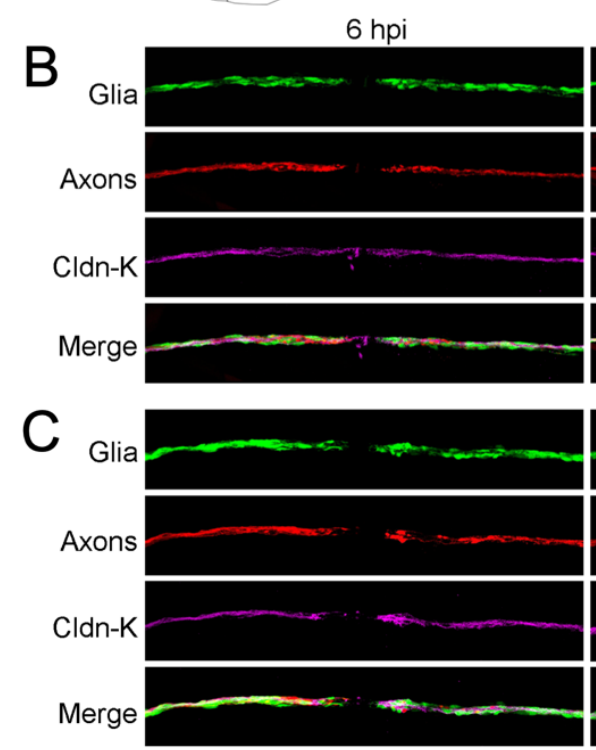

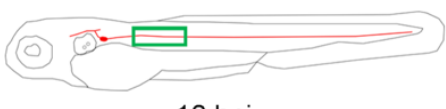

10 hpi
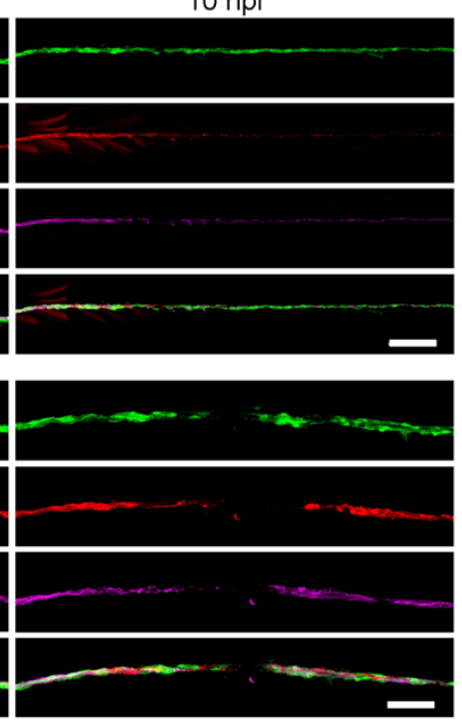
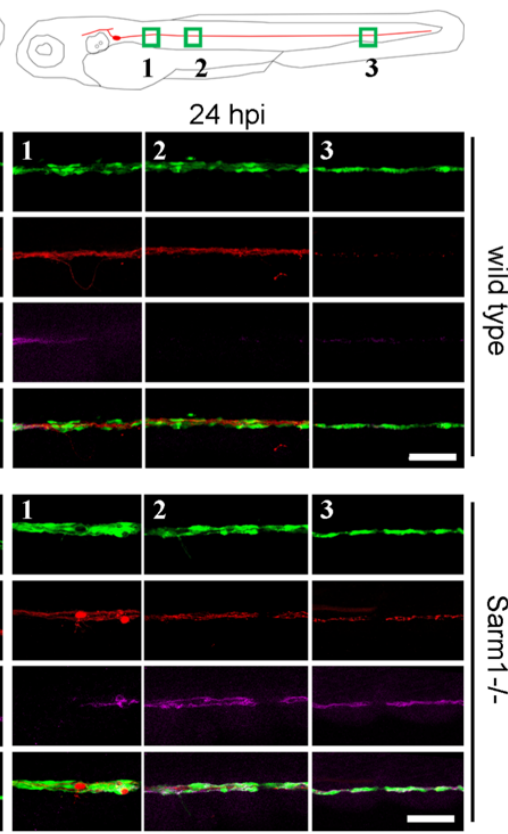

Figure 5. A-C) Schematic model of the confocal imaging locations on severed axons (A). Confocal images of wild type (B) and Sarm 1-/- (C) specimens in 6hpi, 10hpi and 936 24hpi. hpi $=$ hour post injury. The specimens were the 937 Tg[gSAGFF202A;UAS:EGFP; SILL:mCherry] lines and were stained with Claudin-

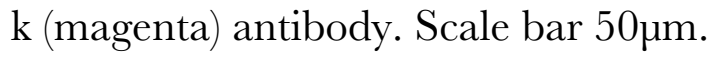



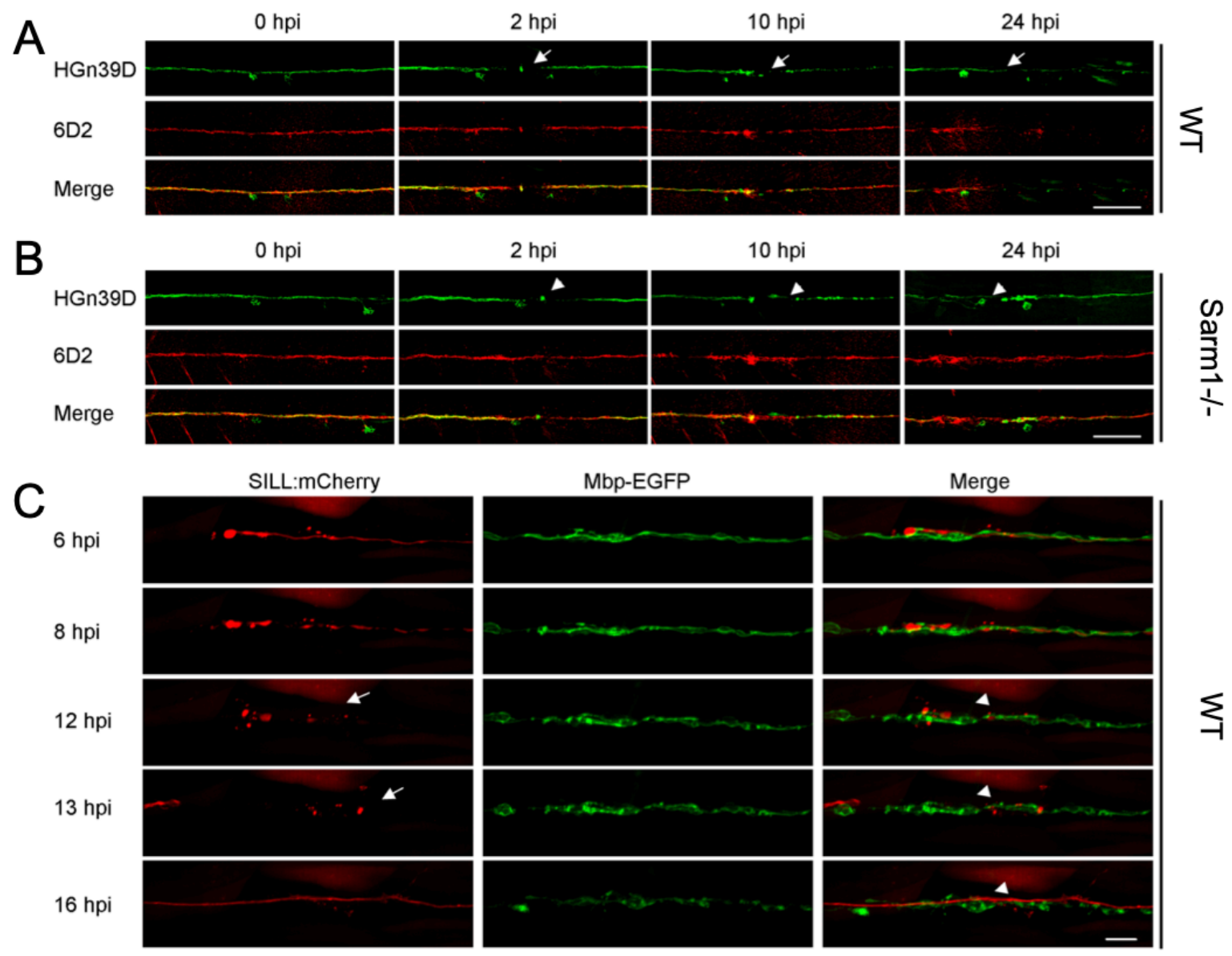

$\sum$
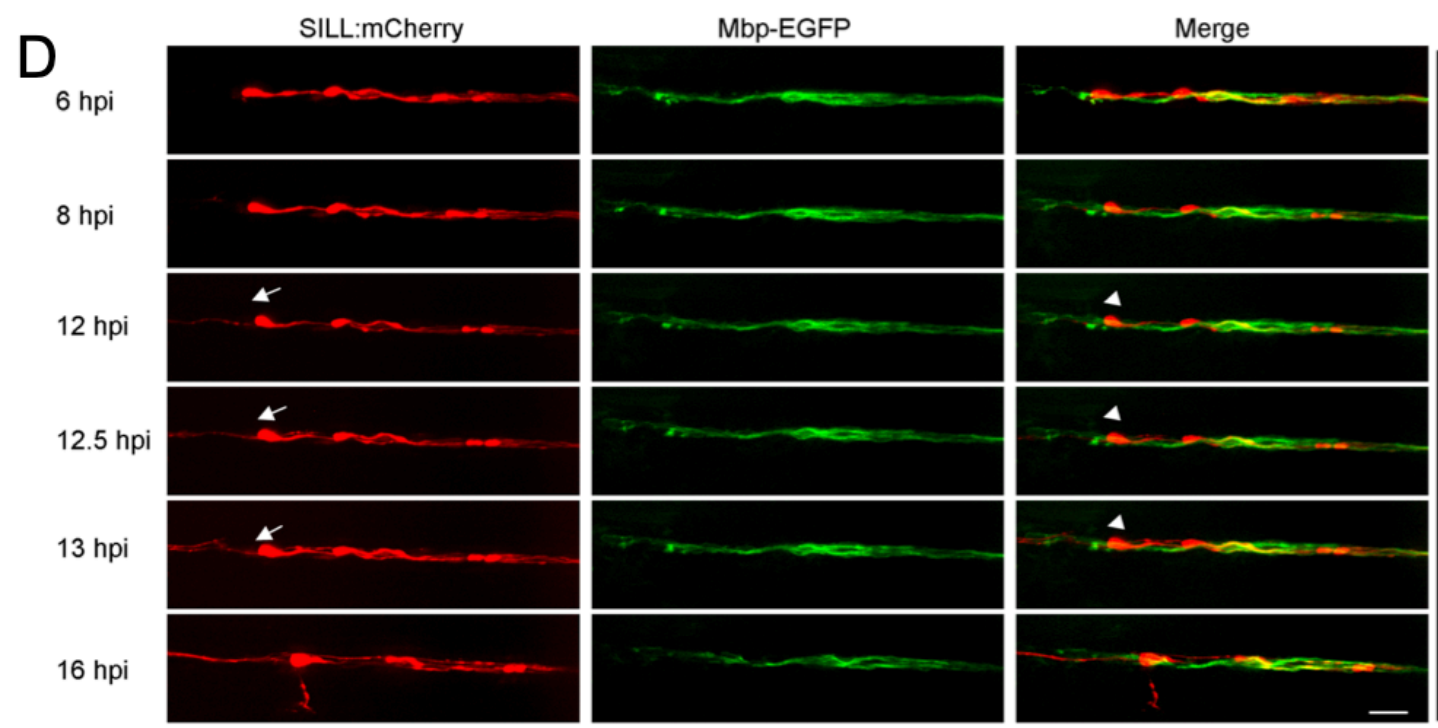

941 Figure 6. A-B) Confocal images of wild type (A) and Sarm1-/- (B) specimens 942 expressing EGFP in sensory neurons of the lateral line (green) and stained with the 943 monoclonal antibody 6D2. Stainings were performed at indicated time points after 
944 axons severing (hpi). The arrows point to the cutting sites. Scale bar is $50 \mu \mathrm{m}$. G) Live

945 imaging of the $\mathrm{Tg}[M b p-E G F P$; SILL:mCherry] after severing. The arrows indicated the

946 fragmented axons and the arrowheads the fragmented myelin. Scale bar $20 \mu \mathrm{m}$. D)

947 Live imaging of the Sarm 1-/- in Tg[Mbp-EGFP; SILL:mCherry] after severing. Arrows

948 indicate regrowing axons, and arrowheads indicate the juxtaposition between the

949 regrowing axons. Scale bar $20 \mu \mathrm{m}$.

950 

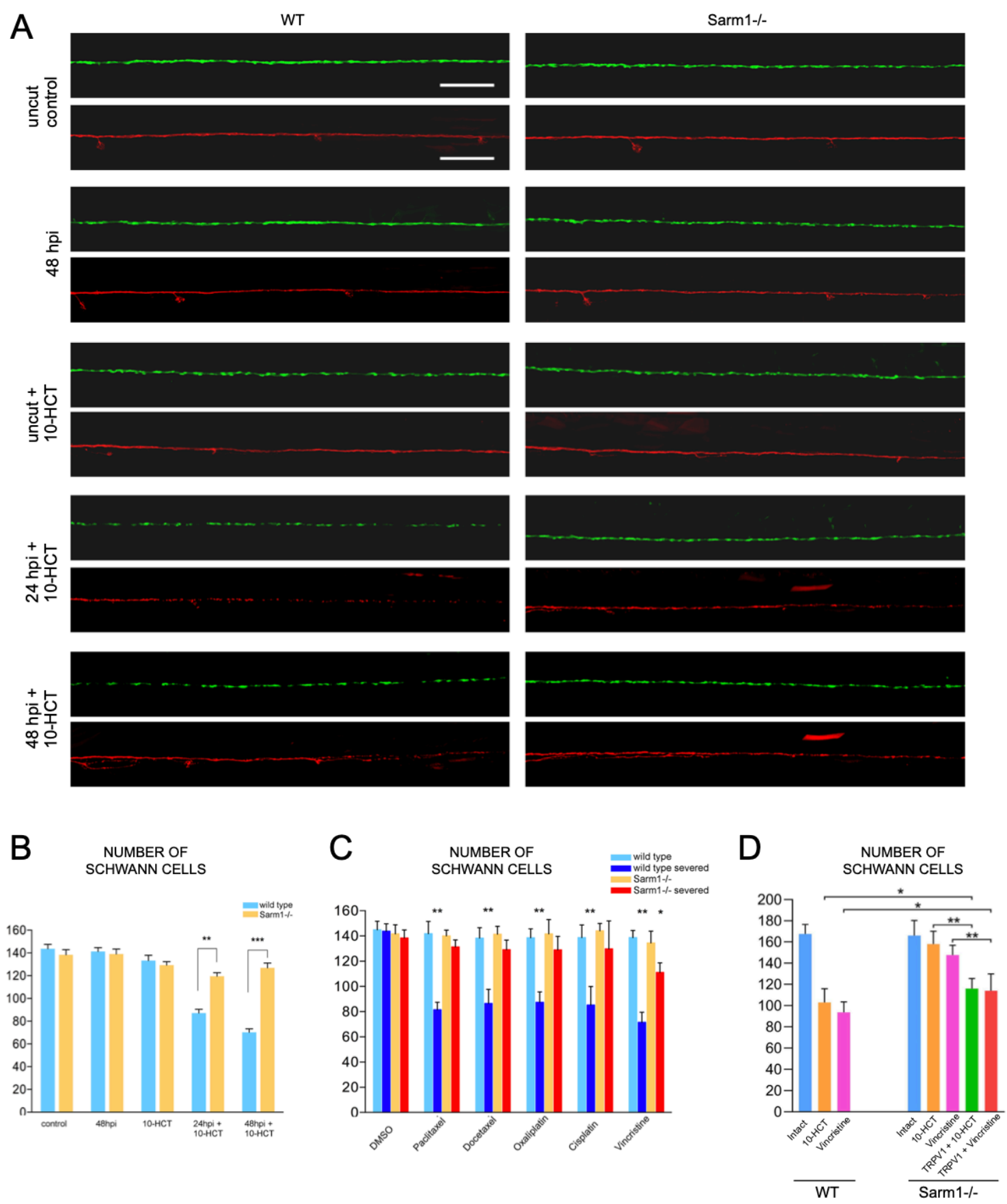

Figure 7. A) Confocal images showing Schwann cells (green) and lateralis sensory axons (red) in a control specimen (in which axons were not transected), in a specimen

95448 hours after axon transection, and in specimens treated with 10-HCT (10955 Hydroxycamptothecin). Left column is wild type and right column shows Sarm1-/-. 956 In all cases, the concentration of 10-HCT in water was $40 \mu \mathrm{m}$. Scale bar $100 \mu \mathrm{m}$. B) 957 Quantification of the Schwann cells from A). Data are shown as mean \pm SEM. ** means $\mathrm{p}<0.01$, one-way ANOVA, $\mathrm{n}=8$ (each group). G) Quantification of 
959 Schwann cells of WT, WT severed, Sarm1-/- and Sarm1-/- severed with the 960 treatment of the indicated chemical compounds for 48 hours. Concentrations:

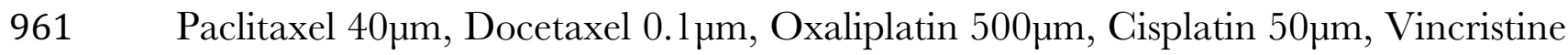
$96250 \mu \mathrm{m}$. Data are shown as mean \pm SEM. * means $\mathrm{p}<0.05$; ** means $\mathrm{p}<0.01$, one963 way ANOVA, $\mathrm{n}=8$ (each group). D) Quantification of Schwann cells after axon 964 severing, in specimens treated with 10-HCT or Vincristine. Left bar group is wild 965 type. Right bar group is Sarm1-/-, and Sarm1-/- with synthetically-eliminated axon 966 segments.

967

968

969

970

971

972

SUPPLEMENTAL MATERIALS 
A
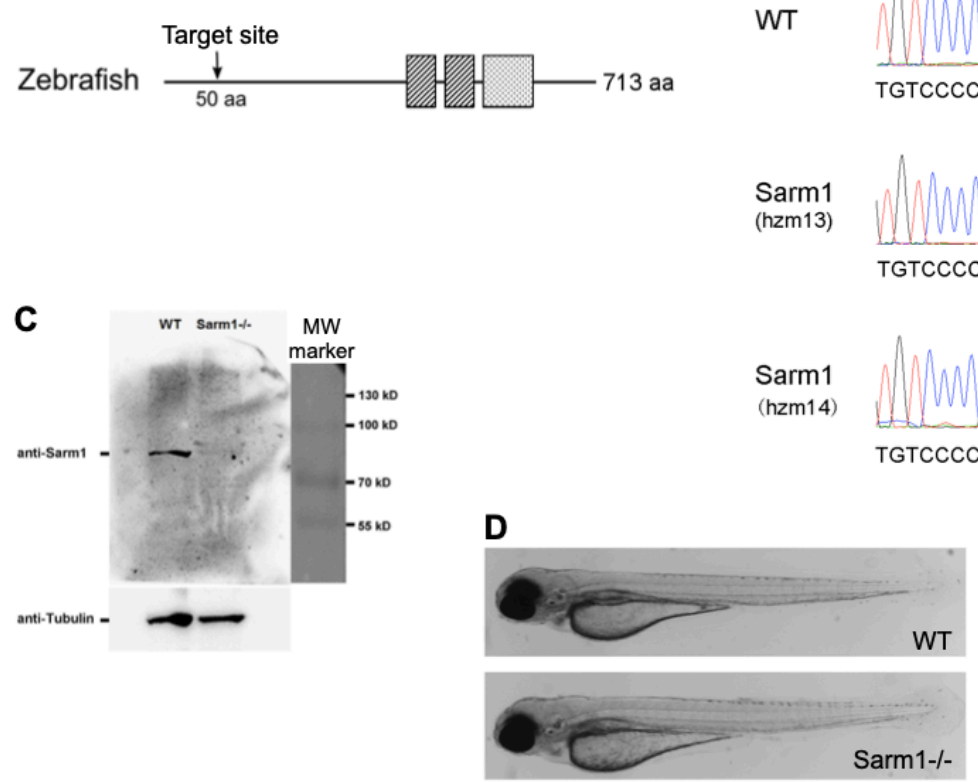

B

WT

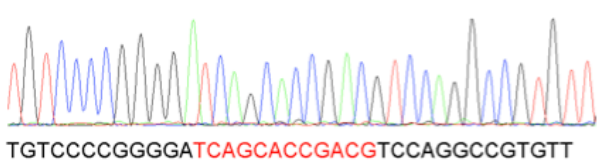

Sarm1 (hzm13)

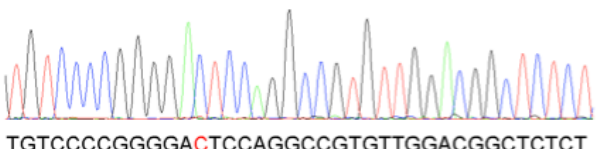

Sarm1 (hzm14)
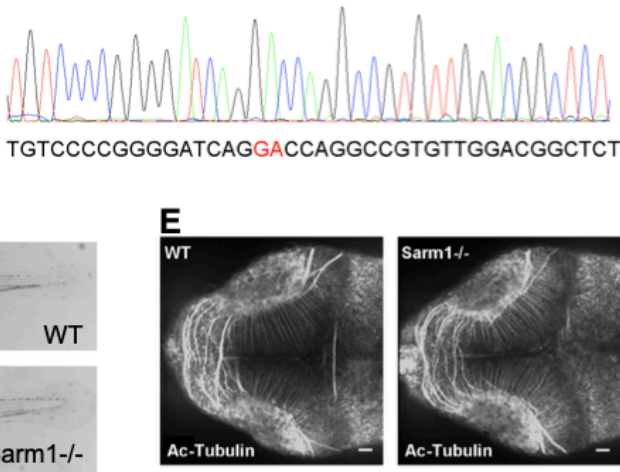

$\mathbf{F}$

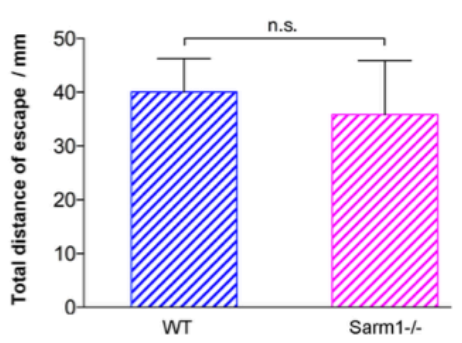

G

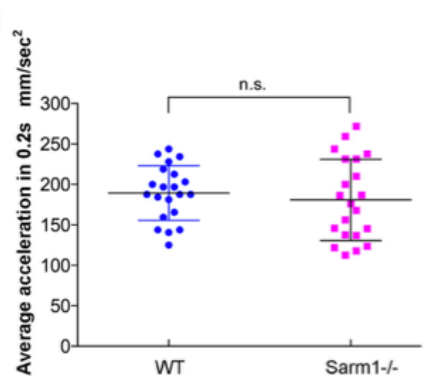

H

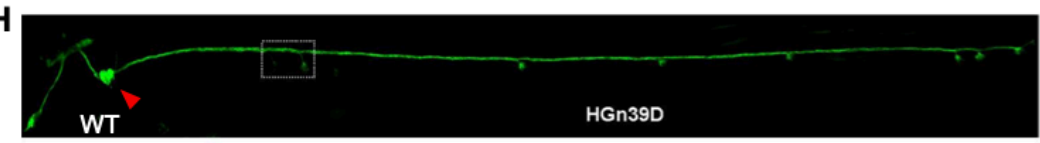

I

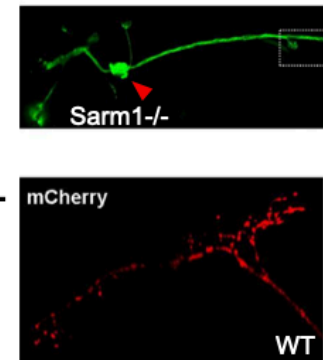

M mcherry

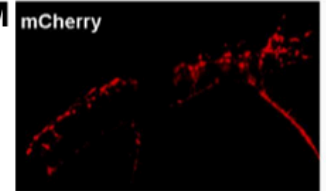

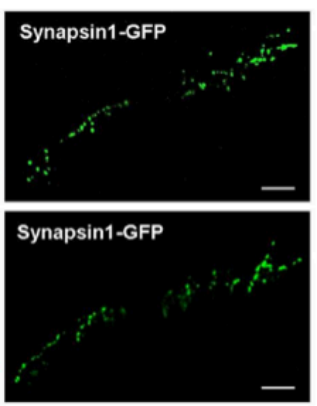

$\mathbf{N}$

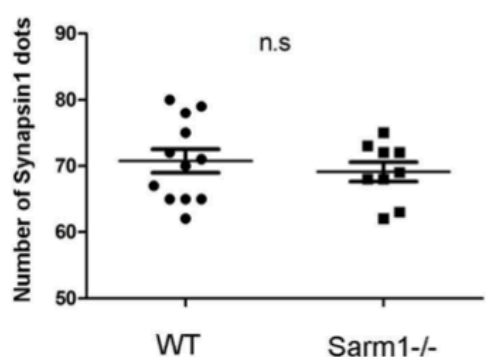

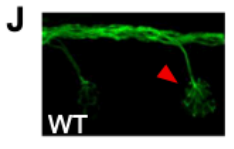

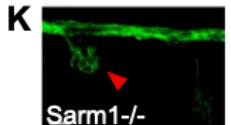

Sarm1-/-

974 Supplemental Figure 1. A) Structure of the zebrafish Sarml protein highlighting

975 the two SAM domains (dark grey), the TIR domain (light grey). The downward arrow indicated the approximate region targeted for mutagenesis, approximately 50 

red the mutagenized area and, below, the two mutant alleles obtained in this study. The hzm13 allele introduces an 11-base deletion and T/C mutation, resulting in a frame shift and premature stop codon. The hzm14 allele is a 7-base deletion and AG/GA mutation that also generates a frame shift and premature stop codon. $\mathbf{G}$ ) Western blot of protein extracts from wild type and Sarm 1 hzm13 fish embryos using a commercial ant-Sarm 1 antibody, revealing absence of the protein in the mutants. An antibody to alpha-Tubulin was used as loading control. D) Low-magnification image of a wild-type 5dpf zebrafish (top) and a homozygous Sarm1 hzm13 (bottom), showing no overall anatomical differences. E) Confocal image of a wild-type 5dpf zebrafish (left) and a homozygous Sarm1 ${ }^{h z m 13}$ (right) stained with an antibody to acetylated Tubulin to mark neurons in the central nervous system, showing no evident defects in the mutants. In this and all figures, rostral is left and caudal is right. Scale bar $20 \mu \mathrm{m}$. F-G) Quantification of sensorimotor function in zebrafish. $(\mathrm{H})$ shows the total distance traveled by larvae after touch-trigger escape response in wild-type (dashed blue bar) and homozygous Sarm1 $1^{h z m 13}$ (dashed magenta bar). (I) dot plot of the average acceleration of wild-type (blue) and homozygous Sarm 1 hzm13 (magenta) after tactile touch-induced escape response. Error bar = SEM; n.s. means no significant difference, Student's t test. wild type n=21, Sarm1-/- n=21. H-I) Confocal image of a 5dpf wild-type $(\mathrm{H})$ and Sarm1-/- (I) larvae carrying the $T g[H G n 39 D]$ transgene to mark lateralis afferent neurons with GFP. The posterior lateral-line ganglion is indicated with a red arrowhead. The dotted box indicates an innervated neuromast (expanded in G). Scale bar $400 \mu \mathrm{m}$. J-K) Confocal image of the peripheral arborization of lateralis neurons in 5dpf wild-type $(\mathrm{H})$ and Sarm 1-/- (I). Red arrows indicate the position of a neuromast from the dotted boxes in (H-I). L-M) Confocal image of the central arborization of lateralis neurons in 5dpf wild-type (L) and Sarm1/- (M), mCherry (red) and Synapsin 1-GFP (green) to reveal normal arborization and pre-synaptic puncta in both cases. N) Quantification of the number of synapsin 1 puncta from $($ E-F), Error bar = SEM; n.s. = not significant, Student's t test. Wild type $\mathrm{n}=15, \operatorname{Sarm} 1-/-\mathrm{n}=15$. 

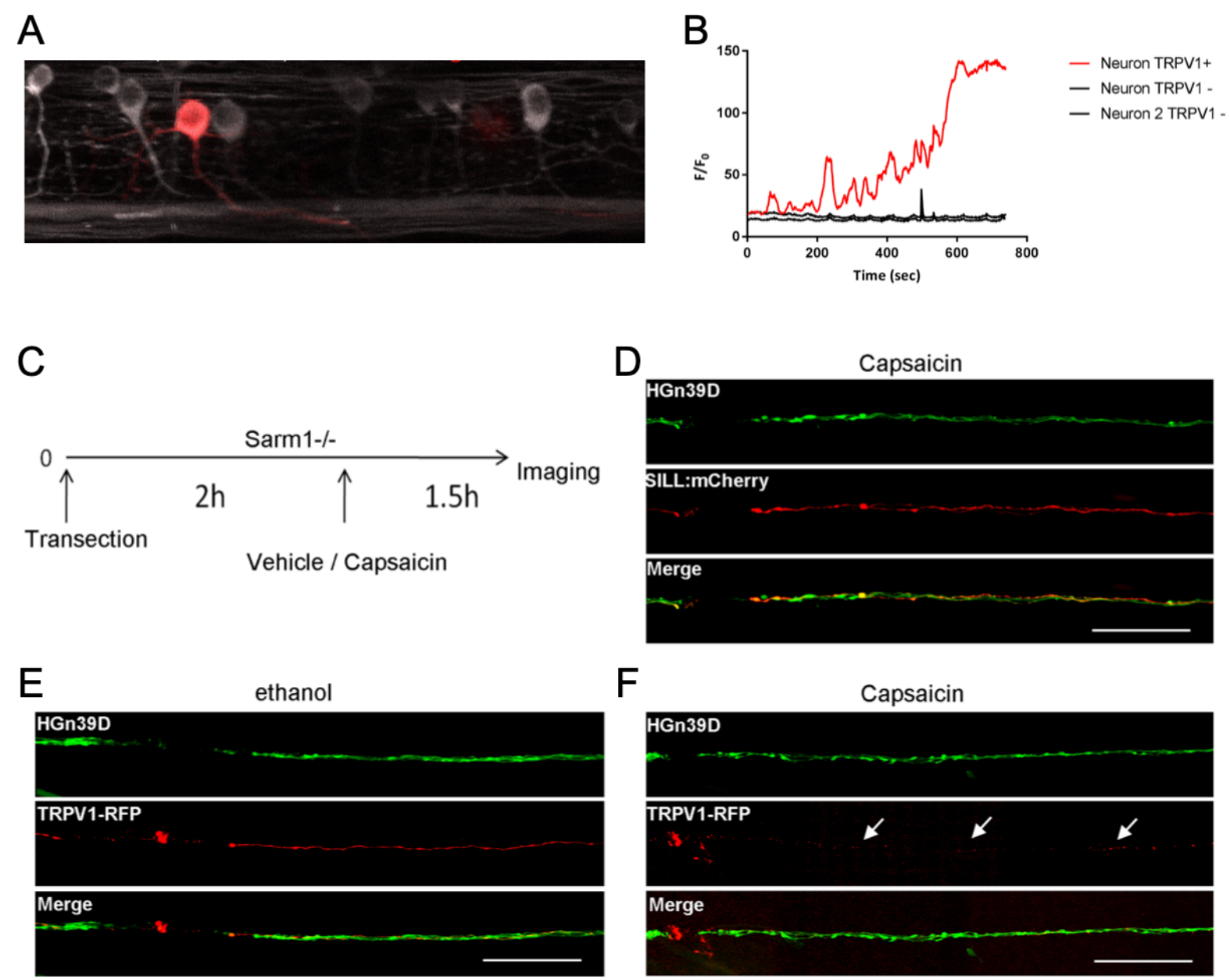

Supplemental Figure 2. A) Confocal image of UAS-TRPV1-tagRFP1 (red,

1010 injected) and UAS-GCaMP (green, transgenic) labeled neurons with Cntnlb:KalTA4. B) Quantification of the calcium signal after capsaicin incubation.

1012 Red plot indicated the TRPV1 positive neuron. The black plots indicated two TRPV1 negative neurons. C) Schematic representation of the experimental strategy

1014 to synthetically elevate calcium in Sarm1-deficient transected axons. Lateralis 1015 sensory neurons were made to express a transgene coding for the rat transient receptor potential cation channel subfamily $\mathrm{V}$ member 1 (TRPV1) fused to RFP, or

1017 simply mCherry. Two hours after axon transection, zebrafish larvae were bathed in 1018 ethanol solution (control), or ethanol containing capsaicin, a natural activator of 1019 TRPV1.90 minutes after treatments, larvae were imaged by confocal microscopy to 1020 assess the extent of distal segment degradation. D) Sarm1-/- fish expressing GFP in all lateralis neurons $(\mathrm{Tg}[\mathrm{HGn39D}])$ and mCherry in a mosaic manner in some neurons. 

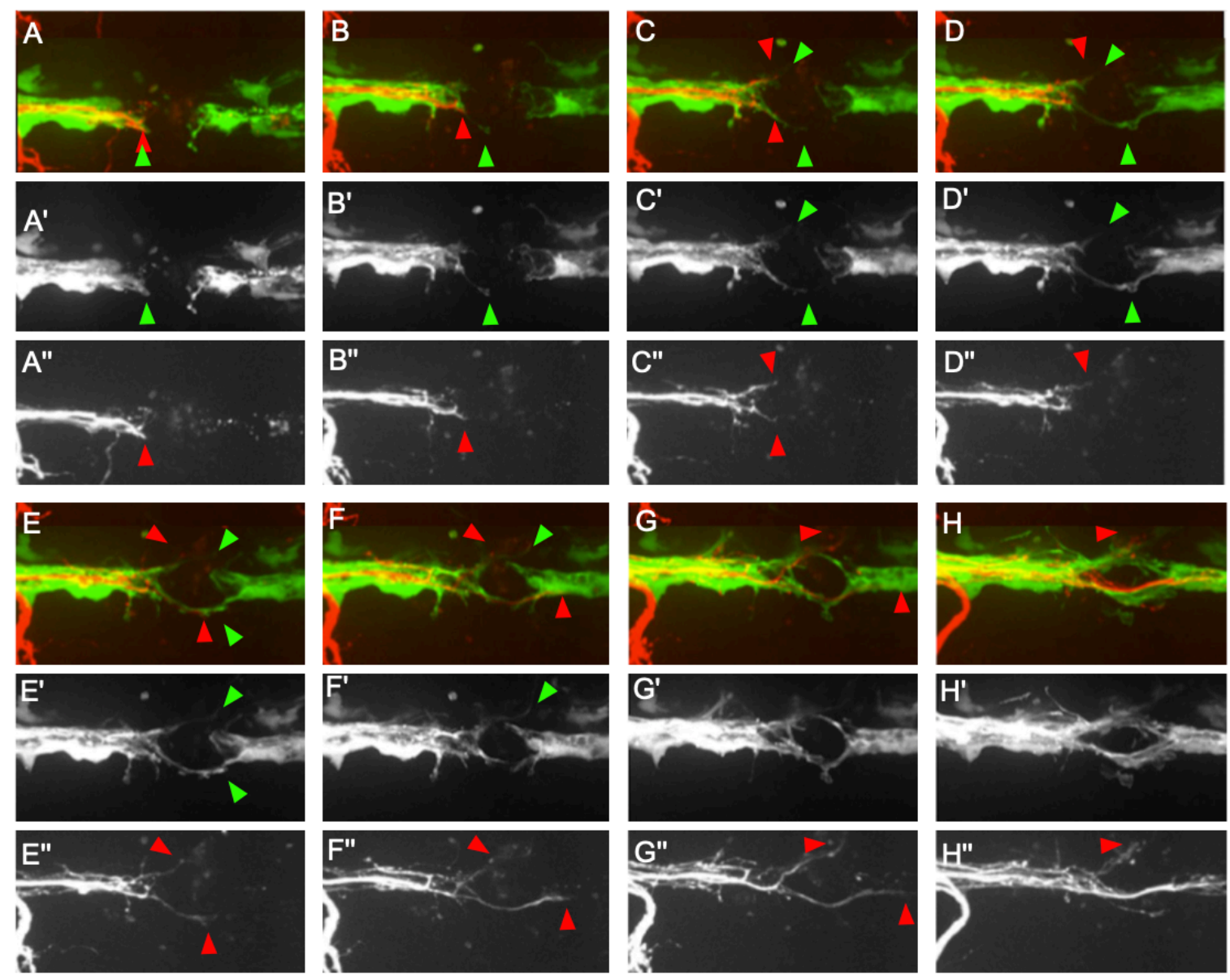

Supplemental Figure 3. Series of still images taken from Supplemental Movie 1 (also shown in Figure 6, panel E). They show eight time points during the repair of the gap in the glial scaffold (A'-H') and axonal regeneration after transection (A'-H') in a wild-type animal. Rostral is left and caudal is right. In all panels, the red arrowheads signal the location of the pioneering growth cone of the regenerating axons. The green arrowheads mark the filopodia-like extensions from Schwann cells adjacent to the glial gap. At the start of the series, the axonal terminal stump and the Schwann cells proximal to the gap co-localize (juxtaposition of the green and red 
arrowhead). In C', a Schwann cell extends a filopodium across the gap, whereas the axons $\left(\mathrm{C}^{\prime \prime}\right)$ do not grow along this extension of across the gap. In D', several extensions from Schwann cells are clearly visible at the top and bottom aspects of the image. The shape of the lower extension from a Schwann cell did not change shape, suggesting that they are stabilized, perhaps through interactions with the substrate. 1042 Proximal axons (D") start to grow along these Schwann-cell protrusions. In E', the 1043 extensions from the anterior and posterior Schwann cells have crossed the gap, 1044 physically interact and commence to reconstitute a continuous glial scaffold. The axonal projections $\left(\mathrm{E}^{\prime \prime}\right)$, however, have suffered a retraction towards the proximal stump. F'-H' show a continuation of Schwann cells behavior, increasing the contacts and closing the gap, which is obvious by the smaller area of the gap (distance between proximal and distal Schwann cells). F"'-H" show a more robust and persistent extension of the axonal growth cones, which grow nearly strictly along the Schwann cells extensions. Although the gap in the glial scaffold is much reduced, nerve fibers 

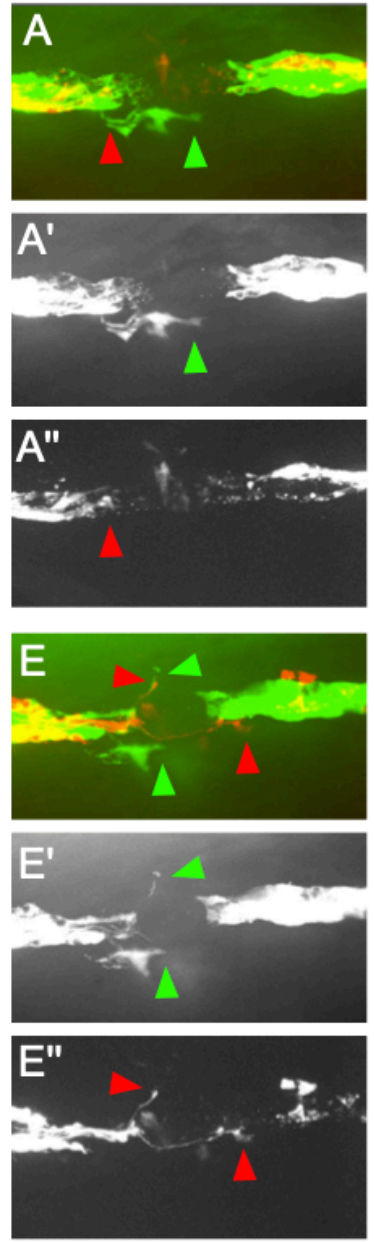
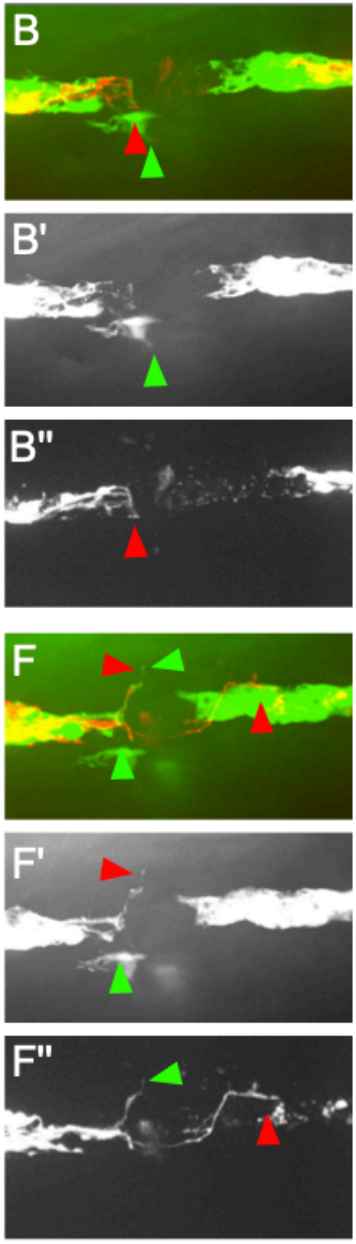
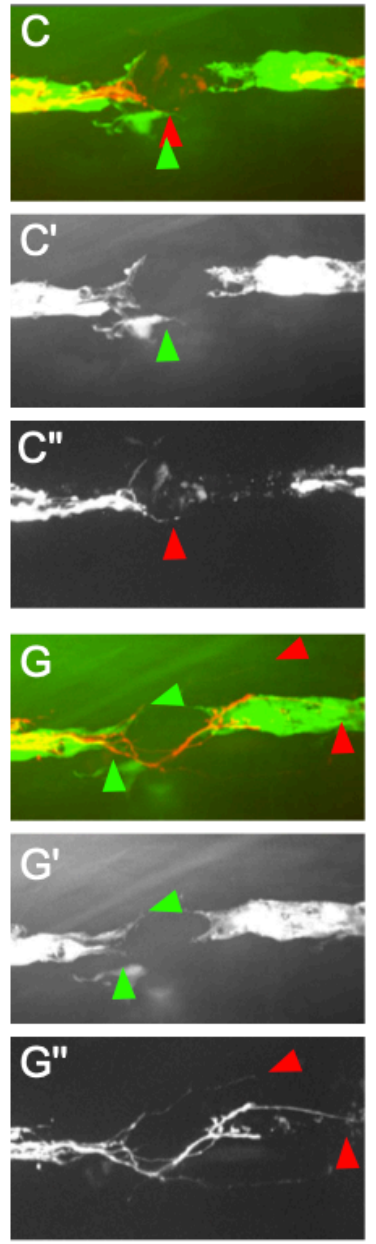
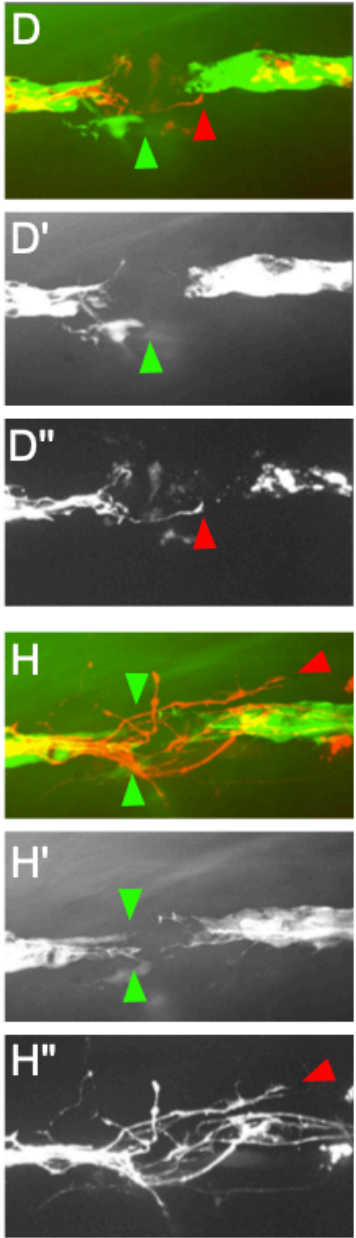

Supplemental Figure 4. Series of still images taken from Supplemental Movie 2 the gap in the Schwann-cell scaffold (A'-H') and axonal regeneration after transection (A"-H"), in a Sarm1-mutant specimen. In all panels, the red arrowheads signal the location of the pioneering growth cone of the regenerating axons, and the green arrowheads mark filopodia-like extensions from Schwann cells. Unlike the wild-type situation shown in Supplemental Figure 1, the Schwann cells adjacent to the gap form small filopodia-like extensions, but which never cross the gap. The proximal axon stumps eventually form growth cones that cross the gap at various locations and, upon finding distal Schwann cells, grow along the glial scaffold (E'-H"). Nerve fibers show extensive local defasciculation (H"). 
1066 Supplemental Movie 1. Videomicroscopic imaging of a double transgenic Tg[SILL:mCherry; Mfap4-memEGFP] 4dpf wild-type specimen in which the mCherry $(+)$ axons were severed by a laser pulse. The video represents for 13 hours of continuous imaging at a 5-minute temporal resolution. Axons (red) and macrophages (green) were visualized relative to the cut (proximal is on the left and distal in on the right).

Supplemental Movie 2. Videomicroscopic imaging of a double transgenic Tg[SILL:mCherry; Mfap4-memEGFP] 4dpf specimen that was mutant for Sarm1, in which the $\mathrm{mCherry}(+)$ axons were severed by a laser pulse. The video represents for 13 hours of continuous imaging at a 5-minute temporal resolution. Axons (red) and macrophages (green) were visualized relative to the cut (proximal is on the left and distal in on the right).

Supplemental Movie 3. Videomicroscopic imaging of a double transgenic Tg[SILL:mCherry; Mfap4-memEGFP] 4dpf specimen that was mutant for Erbb2, in which the mCherry $(+)$ axons were severed by a laser pulse. The video represents for 13 hours of continuous imaging at a 5-minute temporal resolution. Axons (red) and macrophages (green) were visualized relative to the cut (proximal is on the left and distal in on the right). carrying three transgenes Tg[SILL:mCherry; gSAGFF202A; UAS:EGFP], showing the dynamics of axons and Schwann cells after axonal transection. Sensory axons are shown in red and Schwann cells in green. The movie is a maximal projection of confocal stacks. It was recorded during 12 hours at 10-minute intervals. The top panel shows the merged images, the middle panel the Schwann cells, while the bottom panel the axons. 
1095 Supplemental Movie 5. Identical experiment as shown in Supplemental Movie 1, 1096 but conducted in a Sarm1-mutant specimen. It was recorded during 12 hours at 101097 minute intervals. The top panel shows the merged images (SILL:mCherry; 1098 gSAGFF202A; UAS:EGFP), the middle panel the Schwann cells (gSAGFF202A; 1099 UAS:EGFP), and the bottom panel the axons (SILL:mCherry). 